\title{
Aplicación empírica del modelo de Black y Scholes en México: 1991-2000
}

\author{
María Luisa Saavedra García*
}

\section{Resumen}

Esta investigación muestra la aplicación empírica del modelo de Black y Scholes a un conjunto de empresas que cotizan en la Bolsa Mexicana de Valores para saber en qué forma este modelo valúa a estas empresas y para ver si existe algún patrón de comportamiento del modelo, por un periodo de 10 años de 1991 a 2000.

Con el fin de calcular el valor de las empresas con el modelo de Black y Scholes, se utilizó únicamente información pública y se propuso una metodología de aplicación del mismo considerando las limitaciones que esto implicaba y adaptando el modelo al contexto mexicano.

Al contrastar la teoría y las investigaciones antecedentes con los resultados de esta aplicación empírica, encontramos que se validan tanto la teoría como las investigaciones antecedentes, pues los valores arrojados por el modelo resultan ser positivos y elevados, lo cual podría ser interpretado como una sobrevaluación del valor de las empresas si tomamos como patrón de referencia el precio de mercado de las citadas empresas. Es necesario señalar que únicamente se trabajó con las variables contenidas en el modelo sin considerar otros aspectos, como podrían ser variables macroeconómicas o de mercado.

Palabras clave: Black y Scholes, volatilidad, valuación de empresas, valor, precio de mercado, Bolsa Mexicana de Valores

\footnotetext{
* Profesora Investigadora de la Universidad Autónoma del Estado de Hidalgo. Tutora del Programa de Posgrado en Ciencias de la Administración de la Universidad Nacional Autónoma de México.

Correo electrónico: maluisasaavedra@yahoo.com
} 


\section{Introducción}

$\mathrm{E}^{\mathrm{n}}$ ntre los principales modelos de valuación de empresas surge el modelo de valuación de opciones ${ }^{1}$ de Black y Scholes aplicado a la valuación de empresas $^{2}$. Según Merton (1985: 301), la deuda de la empresa es vista como una opción de compra sobre el capital de la misma; de tal manera que si a la fecha de vencimiento de la deuda el valor de la empresa es mayor que la deuda, los acreedores no ejercen su opción; por el contrario, si al vencimiento de la deuda el valor de la empresa es menor que la deuda, los acreedores pueden ejercer su opción de compra y quedarse con la empresa.

Uno de los modelos más conocidos en el tema de valuación de empresas es el de Flujo de efectivo disponible, el cual tiene amplia aplicación tanto en países desarrollados como subdesarrollados con ciertas variantes; sin embargo el modelo de Black y Scholes es uno de los modelos menos conocidos y aplicados para valuar empresas; una de las razones principales es la complejidad de su aplicación y la dificultad para entender su lógica, así como el hecho de que es considerado sólo para valuar opciones.

Por lo anterior nos hacemos la siguiente pregunta de investigación: ¿Cómo puede aplicarse el modelo ${ }^{3}$ de valuación ${ }^{4}$ de opciones de Black y Scholes ${ }^{5}$ aplicado a las empresas que cotizan en la Bolsa Mexicana de Valores y cuál es el resultado de esta valuación?

\footnotetext{
${ }^{1}$ Este modelo aplicado a opciones se puede ver en Abreu (1999), Bellalah y Jacquillat (1995), Díaz (1998), Díaz y Hernández (1998), Galitz (1994), Martínez (1993), Merton (1997) y Rodríguez de Castro (1998).

${ }^{2}$ La aplicación de este modelo a la valuación de empresas puede verse en Adam (1996), Baskshi y Chen (1997), Milla (1998) y Simón (1994).

${ }^{3}$ Según Thierauf (1987: 32), "El modelo es una representación o abstracción de una situación u objeto reales, que muestra las relaciones (directas e indirectas) y las interrelaciones de la acción y la reacción en términos de causa y efecto [...] Una de las razones básicas para el desarrollo de modelos es la de descubrir cuáles son las variables importantes o pertinentes. Este descubrimiento está estrechamente asociado con la investigación de las relaciones que hay entre las variables".

${ }^{4}$ En el contexto de la presente investigación no se considera la teoría del valor, sino sólo el valor muy precisado por una minimización financiera; es decir, el valor se reduce a términos financieros. En este sentido, Van Horne (1993: 102) señala que "el valor para la empresa se crea mediante las inversiones de capital que aprovechan las oportunidades de rendimientos excedentes, aquellas que proporcionan rendimientos en exceso con respecto a los que los mercados financieros requieren para el riesgo supuesto". Por otra parte, Rappaport (1986: 81) indica que las vías para crear valor son el atractivo de la industria y la ventaja competitiva. Por último, Taggart (1994: 69) señala que los determinantes financieros de la creación de valor son el rendimiento sobre el capital, la tasa de crecimiento del capital y el costo de capital. ${ }^{5}$ El modelo de valuación de opciones de Black y Scholes original puede verse en Black y Scholes (1973).
} 
El objetivo de la investigación es proponer una metodología de valuación con el modelo de Black y Scholes y realizar una aplicación empírica del mismo a las empresas que cotizan en la Bolsa Mexicana de Valores con el fin de encontrar si este modelo subestima o sobrestima el valor y si esto se explica por variables vinculadas al modelo o por otros aspectos ${ }^{6}$.

Con respecto a lo anterior se plantea la siguiente hipótesis de investigación:

$\mathrm{H}_{1}$ : Existe una diferencia significativa entre los valores calculados para las empresas que cotizan en la Bolsa Mexicana de Valores de 1991 a 2000, con el modelo Black y Scholes, y el precio de mercado de las mismas de 1991 a 2000.

\section{EI modelo de Black y Scholes}

El modelo de Valuación de Opciones original fue desarrollado por Black y Scholes (1997: 637) para el cálculo del valor de una opción de compra europea ${ }^{7}$ que no paga dividendos; las variables de este modelo son precio de la acción, precio de ejercicio, el tiempo de vencimiento, la varianza del precio de la acción y la tasa libre de riesgo. Merton ${ }^{8}$ modificó el modelo original para incluir el factor de dividendos, que ha sido ampliamente aplicado para calcular opciones de empresas que pagan dividendos y que muchos investigadores han comprobado su utilidad.

Bellalah y Jacquillat (1995: 625) en sus investigaciones comprueban que no existe diferencia significativa entre el precio de la opción calculado con el modelo y el valor de mercado de la misma; así determinan la validez del modelo. En este sentido, Abreu (1999: 133) coincide en señalar que es un modelo de menor sesgo estadístico y más eficiente que otros. Sin embargo, otros investigadores han descubierto que el modelo tiene sus debilidades; por ejemplo, Simons (1997: 25) señala que se puede sobrestimar el valor dado que considera que los rendimientos de los valores se distribuyen normalmente; asimismo, para la aplicación del modelo se debe conocer la volatilidad de la acción, a través de una estimación estadística que puede estar sujeta a errores.

\footnotetext{
${ }^{6}$ Los otros aspectos podrían ser las características del sector, el comportamiento de las variables del entorno económico, etc., los cuales no serán desarrollados en la presente investigación.

${ }^{7}$ Otorga el derecho, pero no la obligación, de comprar un activo a un precio estipulado y puede ser ejercida únicamente en la fecha de expiración.

${ }^{8}$ Citado en Coller (1997: 27).
} 
Leslie y Michaels (1997: 20), Luehrman (Jul.1998: 98), Copeland y Keenant (1998: 140) y Alzugaray (2000: 112), analizan la aplicación del modelo de opciones a la evaluación de inversiones de largo plazo en la empresa, concluyendo que conduce a la administración hacia la maximización de oportunidades mientras minimiza los riesgos, asegurándose que la empresa vea cada situación como una inversión inicial contra una posibilidad futura.

Desde este punto de vista, la opción no sólo se puede ejercer en la fecha de vencimiento, sino que se plantea una gama de posibilidades donde se puede determinar el momento más adecuado para tomar la decisión de inversión. Se puede examinar un rango completo de oportunidades cambiantes y tomar decisiones en el largo plazo. Esto se puede aplicar así porque, según Luerhman (1998b: 34), en términos financieros una estrategia de negocios es mucho más semejante a una serie de opciones que a una serie de flujos de efectivo estáticos; sin embargo, López (2001: 5) señala que la flexibilidad también puede incorporarse en los flujos de efectivo a través del análisis de escenarios alternativos y cambiantes. Esto trae como consecuencia que valorar una inversión introduciendo opciones reales - como abandono, crecimiento, retraso o cambio en la utilización de activos para introducir flexibilidad en el análisis - es equivalente a valorar ese proyecto utilizando escenarios alternativos con probabilidades.

El modelo de valuación de opciones de Black y Scholes aplicado a la valuación de empresas se deriva del análisis contingente de Merton (1985: 37), donde sostiene que las deudas corporativas en general pueden ser vistas como simples contratos de opciones, determinando entonces que el modelo de opciones se puede utilizar para valuar acciones. Stewart (1996: 100), coincide al señalar que las acciones comunes son opciones de compra, que toman (o retienen) los activos de la empresa para pagar su deuda. Los accionistas pueden vender los activos de la firma a sus acreedores; el precio de ejercicio de la venta sería el valor de la deuda. En este mismo sentido, Damodaran (1994: 346) muestra la aplicación de este modelo a la valuación de la empresa; el valor de la empresa, señala este autor, dependerá del valor del activo, del valor del pasivo a futuro, del tiempo de vencimiento de la deuda, de la volatilidad, es decir, del riesgo del activo y de la tasa libre de riesgo. Este modelo se considera de gran utilidad para valuar empresas que trabajan con alto apalancamiento. También, Adam (1996: 42) realizó un análisis de la aplicación de este modelo comparándolo con los modelos de Valor en Libros, Precio-Beneficio y Valor de Mercado, para llegar a la conclusión de que el Black y Scholes es el más confiable y completo. 
Luherman (1997a: 137) corrobora lo anterior al señalar que la clave para valuar a la empresa como una opción se encuentra en la capacidad de discernir una simple correspondencia, entre las características de la empresa y las de una opción. De este modo, el valor de los activos operativos es semejante al precio de la acción; el período que la empresa espera antes de tomar una decisión es semejante al tiempo de expiración de la opción de compra; la incertidumbre acerca del valor de los activos operativos es capturado por la varianza de los retornos, y es análoga a la varianza de los retornos de la acción. Sin embargo también señala que no es muy conveniente valuar acciones con este modelo, puesto que las acciones apalancadas son una secuencia de opciones relativas, incluyendo opciones sobre opciones (debido a que la deuda se paga y se vuelve a contraer). A este respecto, Mascareñas (2000: 385) propone una solución que consiste en calcular el valor y el plazo de una emisión de bonos cupón cero ${ }^{9}$ que fuera equivalente a todas las emisiones de deuda que actualmente tiene vigentes la empresa. Esto se realiza a través del cálculo de la duración, la cual se define como la media ponderada de los vencimientos de los flujos de caja ponderados por su valor actual.

Este modelo es el menos usado por su complejidad; sin embargo, consideramos que la interrelación de sus variables pudiera llevar a una valuación más real de las empresas con alto apalancamiento, pues considera su nivel de riesgo.

Como mencionamos, el modelo de Black y Scholes aplicado a la valuación de empresas se basa en el análisis contingente, el cual se explica a continuación.

\subsection{El análisis contingente}

El análisis contingente es una técnica para determinar el precio de un valor cuyo resultado depende del precio de uno o más valores El origen del análisis contingente es el modelo de opciones de Black y Scholes, el cual contiene elementos cualitativos con un gran significado práctico. Esta teoría sostiene que las deudas corporativas, en general, pueden ser vistas como combinaciones de simples contratos de opciones (Merton, 1985: 301).

El análisis contingente provee un marco de trabajo unificado donde se puede analizar la estructura de las deudas corporativas e implica que el modelo de opciones

\footnotetext{
${ }_{9}$ Título que no paga intereses durante su vida, sino que lo hace íntegramente en el momento en el que se amortiza el capital.
} 
puede ser usado para determinar el precio de las acciones. Este modelo generalizado es el fundamento del análisis contingente.

\subsubsection{Los pasivos corporativos como opciones}

El análisis contingente establece una correspondencia entre las opciones y las deudas corporativas. Para ello considere la hoja de balance de la firma "La Moderna" (cuadro No 1); esta empresa tiene sólo dos fuentes de financiamiento: capital (C) y una deuda cupón cero (D), donde el capital no percibe dividendos y la empresa no puede emitir nuevos valores además de la deuda pendiente.

\section{Cuadro 1}

\section{Balance de la empresa "La Moderna"}

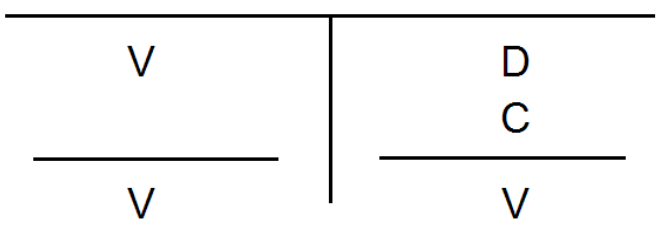

El lado izquierdo del balance representa el valor económico (V), de la empresa. El lado derecho representa el valor económico de todas las deudas de la empresa.

Las gráficas 1 y 2 describen el valor del capital y la deuda con riesgo como dependientes del valor de la empresa a la fecha de vencimiento de la deuda. En la gráfica 1 se ilustra que si a la fecha de vencimiento de la deuda el valor de la empresa es más grande que el principal $(\mathrm{P}), \mathrm{V}>\mathrm{P}$, entonces la deuda puede ser pagada, $\mathrm{D}=\mathrm{P}$, y el capital será $\mathrm{V}-\mathrm{P}$.

\section{Gráfica 1}

\section{Estructura de pago de capital}

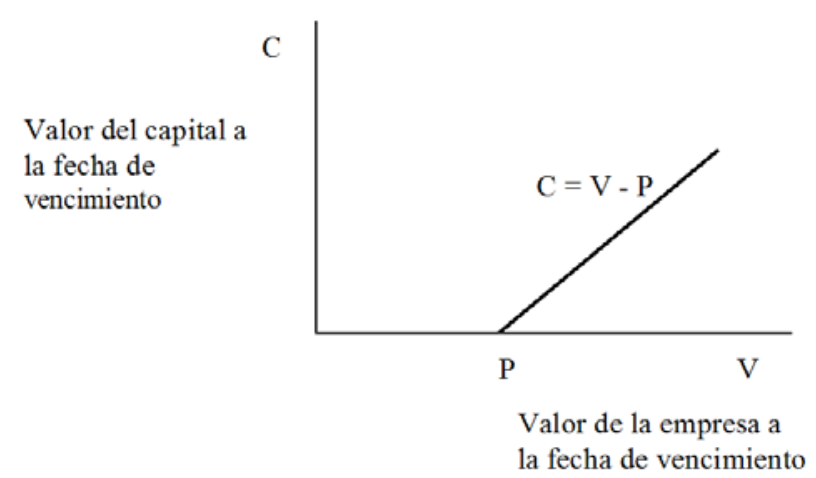


Por otro lado, en la gráfica 2 se muestra que si a la fecha de vencimiento de la deuda el valor de la empresa es menor que el principal, $\mathrm{V}<\mathrm{P}$, entonces el capital puede perder su valor, $\mathrm{C}=0$, y es preferible entregar la empresa a los acreedores que pagar la deuda. En este caso, $\mathrm{D}=\mathrm{V}$.

\section{Gráfica 2}

\section{Estructura de pago de deuda}

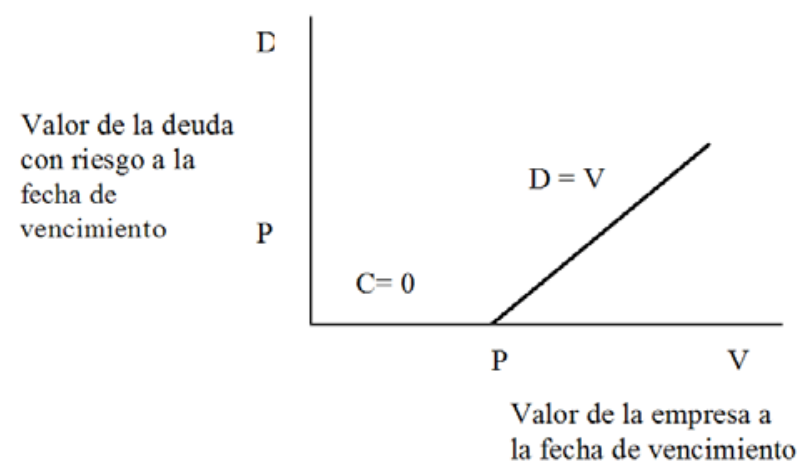

Así, a la fecha de vencimiento de la deuda el valor del capital puede ser representado de la siguiente forma:

$$
\mathbf{C}=\text { máx }(\mathbf{V}-\mathbf{P}, \mathbf{0})
$$

La expresión anterior indica que el valor del capital de la firma a la fecha de vencimiento de la deuda es la diferencia máxima entre el valor de la firma y el pago del principal y cero.

El valor de la deuda con riesgo D a la fecha de vencimiento se puede representar como:

$$
\mathrm{D}=\min (\mathrm{V}, \mathrm{P})
$$

La expresión anterior indica que el valor de la deuda con riesgo a la fecha de vencimiento es el mínimo de V y P; ambos — capital y deuda con riesgo — son valores contingentes cuyo valor es contingente sobre el valor de la empresa. 
El capital y la presencia de un bono cupón cero ${ }^{10}$ de deuda con riesgo son directamente análogos a una opción de compra europea suscrita sobre el valor de la firma (V) con un precio de ejercicio (P) igual al pago del principal de la deuda y a una fecha de expiración igual a la fecha de vencimiento de la deuda. De este modo, el capital puede ser visto como una opción de compra con derecho a comprar la firma por P pesos; es decir, el precio de compra será el valor de la deuda que tiene la empresa en ese momento.

\subsection{El valor de la empresa}

En la caracterización de las deudas corporativas, como las opciones, el valor de la empresa es el activo subyacente sobre el que son suscritas dichas opciones; la promesa de deuda principal $\mathrm{P}$ es el precio de ejercicio; $\mathrm{y}$ la fecha de vencimiento de la deuda es la fecha de expiración de la opción (Merton, 1985: 304).

De aquí parte la analogía para determinar el valor de la empresa con el modelo de valuación de opciones de Black y Scholes, la fórmula se expresa de la siguiente manera (Damodaran, 1994: 346):

$$
C=S \quad N\left(d_{1}\right)-X e^{-r T} \mathbf{N}\left(d_{2}\right)
$$

\section{Donde:}

$$
\begin{gathered}
\mathbf{d}_{1}=\operatorname{Ln}(\mathbf{S} / \mathbf{X})+\left(\mathbf{r}+\underline{\sigma^{2} / 2}\right) \mathbf{T} \\
\sigma \sqrt{ } \mathrm{T} \\
\mathbf{d}_{2}=\mathbf{d}_{1}-\sigma \sqrt{ } \mathbf{T}
\end{gathered}
$$

${ }_{10}$ Título que no paga intereses durante su vida, sino que lo hace íntegramente en el momento en el que se amortiza el capital. 
Tabla 1

Analogía de los valores de la fórmula de Black y Scholes

\begin{tabular}{|c|c|c|}
\hline Variable & Valuación de opciones & Valuación de empresas \\
\hline $\mathbf{C}$ & Precio de la opción & Valor de la empresa \\
\hline $\mathbf{S}$ & Precio del activo subyacente & $\begin{array}{c}\text { Valor presente de los flujos } \\
\text { de efectivo }\end{array}$ \\
\hline $\mathbf{X}$ & Precio de ejercicio & Valor futuro de la deuda \\
\hline $\mathbf{r}$ & Tasa de interés & Tasa libre de riesgo \\
\hline $\boldsymbol{\sigma}$ & Volatilidad del precio del & $\begin{array}{c}\text { Volatilidad de los flujos de } \\
\text { efectivo o de las acciones }\end{array}$ \\
\hline $\mathbf{T}$ & Tiempo hasta vencimiento & Tiempo de la proyección \\
\hline
\end{tabular}

\subsubsection{El modelo de valuación de opciones de Black y Scholes aplicado a la valuación de empresas en el contexto mexicano}

En 1994, la doctora Simón (1994: 249) calculó el valor de AHMSA ${ }^{11}$ con el modelo de Black y Scholes, utilizando la fórmula de este modelo y considerando lo siguiente:

$\mathbf{C}=$ Valor de la empresa

$\mathbf{S}=$ Valor actual de los activos a $1991=1,971 \mathrm{mdd}$.

$\mathbf{X}=$ Valor actual del pasivo a $1991=625 \mathrm{mdd}$.

$\mathbf{r}=$ Tasa libre de riesgo Cetes a 28 días $=12 \%$

$\sigma=$ Desviación estándar de la tasa anual de rendimiento de la acción o volatilidad de la acción $=15 \%$

$\mathbf{T}=$ Vencimiento de la acción $=3$ años, es decir a 1994.

El valor de la opción a 1991, según este modelo, resultó ser de 1,553 mdd, lo cual representa lo que vale la opción de compra de la empresa. Dado que el capital contable a la fecha de la venta era de 1,346 mdd., el valor de la opción de compra es mayor en 187 mdd. La investigadora llegó a la conclusión de que el valor de la

${ }^{11}$ Altos Hornos de México, S.A. de C.V. 
opción calculado con la fórmula de Black y Scholes es independiente del rendimiento esperado de la inversión; asimismo, se puede concluir que el precio de venta ( $10 \%$ de su capital contable) fue menor que lo que realmente valía AHMSA al calcular el valor con este modelo.

Esta investigación sentó las bases en México para considerar este modelo como una alternativa para valuar a las empresas mexicanas; sin embargo, su aplicación fue estática considerando los datos de un sólo año, lo cual limita la aplicación del modelo ignorando el potencial de crecimiento de la compañía; por otra parte, considera el valor actual del pasivo cuando la teoría de opciones señala que se debe usar el pasivo a vencimiento. Esto último podría haber llevado a un error de sobrevaluación en el cálculo del valor de AHMSA dado que se usó un valor de pasivo más bajo.

En otra investigación, Milla (1998: 126) usó la adaptación del Modelo de Black y Scholes, tal como lo propone Luehrman (1998a: 95), para determinar el valor de la empresa AHMSA, dicho modelo considera las siguientes variables:

$$
\begin{aligned}
& \mathrm{C}=\text { Valor de la opción de compra de AHMSA } \\
& \mathrm{S}=\text { Valor presente de los flujos futuros reales de AHMSA, } \\
& \mathrm{X}=\text { Valor futuro de la deuda como bono cupón cero, usando } \\
& \text { una tasa de } 51.21 \%=\$ 622^{\prime} 290,110 \\
& \mathrm{r}=\text { Cetes a } 91 \text { días } \\
& \mathrm{s} \quad=\quad \text { Volatilidad del precio de la acción }=31.57 \% \\
& \mathrm{~T}=\text { Período de la proyección }=7 \text { años }
\end{aligned}
$$

Después de aplicar el modelo, los resultados obtenidos fueron:

$\mathrm{C}=\$ 15^{\prime} 170,000$ entre el número de acciones $388,375=39.06$

Este valor intrínseco es superior al precio de mercado al 31 de diciembre de 1996, que era $\$ 16.50$ pesos.

Se llega a la conclusión de que la diferencia entre el precio teórico y el de mercado se debe al rezago que éste tiene sobre todo, en el nivel de apalancamiento y en el impacto de las variables macroeconómicas a los que es muy susceptible AHMSA. 
Esta investigación incorpora un enfoque muy interesante al considerar como valor del activo subyacente al valor presente de los flujos de efectivo reales de AHMSA calculados con el modelo FED; esto en función de la aplicación de la teoría propuesta por Luehrman (1998a: 95). Sin embargo, vemos que el resultado es una sobrevaluación de la acción que, independientemente de las razones que se le atribuyen, se debería investigar si es la tendencia del modelo cuando las empresas tienen alto apalancamiento como en este caso.

\subsection{Ventajas y desventajas del modelo de valuación de opciones de Black y Scholes}

\section{Ventajas:}

- Permite introducir en la valuación un factor de riesgo congruente con el comportamiento de la acción en el Mercado de Valores, por lo tanto identifican el riesgo sistemático.

- Es muy útil para valuar empresas que trabajan con alto apalancamiento porque considera esta variable dentro del modelo.

\section{Desventajas:}

- Los supuestos de una varianza constante y dividendos no pueden ser sostenidos cuando se calcula el valor en el largo plazo (Damodaran, 1994:19).

- Es un modelo complejo y difícil de comprender.

- No es significativo cuando se aplica a empresas con bajo apalancamiento financiero.

A pesar de las desventajas del modelo y la complejidad de su aplicación, las investigaciones previas han demostrado que es un modelo muy útil y que es viable su aplicación a las empresas que operan en el entorno mexicano, pues su característica es de ser altamente volátil. 


\section{Metodología del cálculo del modelo de Black y Scholes para determinar el valor de la empresa}

\subsection{Variables del modelo}

De acuerdo con la metodología de este modelo aplicado por Simón (1994: 249), las variables de este modelo según su fórmula de aplicación son:

C = Valor de la empresa, se calculará con las siguientes variables:

a. $\mathrm{S} \quad=$ Valor actual de los activos

b. $\mathrm{X}=$ Valor actual del pasivo

c. $\mathrm{r}=$ Tasa libre de riesgo, tasa de Cetes anualizada a 28 días

d. $\sigma=$ Desviación estándar de la tasa anual de rendimiento de la acción o volatilidad de la acción

e. $\mathrm{T}=$ Tiempo hasta el vencimiento/vencimiento de la deuda

f. $\mathrm{e}=$ Es la constante matemática aproximada por 2.71828

g. $\operatorname{Ln}=$ Logaritmo natural

h. $N\left(d_{t}\right)=$ Función de densidad del área bajo la curva normal estándar

\subsection{Medición de las variables}

Específicamente para el cálculo del valor al aplicar el modelo del Black y Scholes a las empresas que cotizan en la Bolsa Mexicana de Valores, las variables del mismo se medirán así:

a. $\mathbf{S}=$ Valor actual de los activos.

Indicador : miles de pesos

Se tomará el valor del activo total que aparece en el Estado de Situación Financiera de la empresa.

b. $\mathbf{X}=$ Valor actual del pasivo.

Indicador : miles de pesos 
El modelo de valuación de opciones considera al pasivo como un bono cupón cero tal como lo explican Merton y Mason (1985:34); por esa razón no se tomará el valor actual del pasivo como señala la metodología propuesta por Simón (1994: 250), sino el valor del pasivo a futuro, el cual se calculará usando la tasa de costo de pasivo para cada empresa. Se considerará un plazo de vencimiento de 3 años ${ }^{12}$.

Para lo anterior se usará la fórmula de interés compuesto: $M=C(1+i)^{n}$

$$
\begin{aligned}
& \text { Donde: } \mathrm{M}=\text { Valor del pasivo a futuro } \\
& \mathrm{C}=\text { Valor actual de la deuda } \\
& \mathrm{i}=\text { Costo del pasivo } \\
& \mathrm{n}=\text { Plazo de vencimiento de la deuda en años } \\
& \text { c. } r=\text { Tasa libre de riesgo. } \\
& \text { Indicador: porcentaje }
\end{aligned}
$$

Se usará como referencia la tasa anualizada de Cetes a 28 días ${ }^{13}$, quitándole el efecto inflacionario, puesto que los datos de la información financiera con la que se trabajará están expresados en pesos constantes.

La tasa real libre de riesgo se determinará así:

$$
(1+\text { Tasa nominal de Cetes } / 1+\text { Tasa de inflación })-1
$$

d. $\sigma=$ Desviación estándar de la tasa anual de rendimiento de la acción o volatilidad de la acción. Indicador : porcentaje

\footnotetext{
${ }^{12}$ Se considera este plazo de vencimiento como un promedio del plazo de endeudamiento de las empresas mexicanas que cotizan en la BMV, considerando que las mismas no se endeudan en el largo plazo.

${ }^{13}$ Se usará la tasa de Cetes a 28 días, puesto que es la tasa que pagan los valores gubernamentales en México, además de que es la tasa de referencia más usada por los expertos en cuanto proyección de variables macroeconómicas se refiere.
} 
Se usará el dato de la volatilidad anual ${ }^{14}$ de la acción de la empresa, el cual aparece en el Anuario bursátil que publica la Bolsa Mexicana de Valores, cada año.

e. $\mathrm{T}=$ Vencimiento de la deuda.

Indicador : años

Se considerará un período de vencimiento de la deuda de tres años, puesto que el promedio de las empresas mexicanas no se endeuda a largo plazo, sino más bien contraen deuda a mediano plazo.

$\mathbf{C}=$ Valor de la empresa. Se determinará con las variables anteriores aplicando la siguiente fórmula:

Indicador: miles de pesos

$$
C=S \quad N\left(d_{1}\right)-X \quad e^{-r T} N\left(d_{2}\right)
$$

Donde:

$$
\begin{gathered}
\mathrm{d}_{1}=\operatorname{Ln}(\mathrm{S} / \mathrm{X})+\left(\mathrm{r}+\sigma^{2} / 2\right) \mathrm{T} \\
\sigma \sqrt{ } \mathbf{T} \\
\mathrm{d}_{2}=\mathrm{d}_{1}-\sigma \sqrt{ } \mathbf{T}
\end{gathered}
$$

\section{Determinación de la muestra}

Para conformar la muestra se obtuvieron datos de 71 empresas que cotizan en la Bolsa Mexicana de Valores; esta información proviene de las bases de datos de la propia BMV y de la Comisión Nacional Bancaria y de Valores para el caso de los bancos. Se consideró un período de estudio de 10 años (1991 a 2000) para reflejar el impacto de los cambios sexenales en la economía, así también para simplificar los cálculos se consideraron datos anuales.

\footnotetext{
${ }^{14}$ Se tomará la volatilidad anual de la acción con el fin de simplificar los cálculos, puesto que la muestra es de 71 empresas a las que se les tiene que calcular el valor con Black y Scholes para cada uno de los diez años de 1991 a 2000.
} 
La clasificación de las empresas por sectores se hizo de acuerdo con el sistema que se usa en el medio bursátil (ver anexo No 1). Se separó en tres partes a las empresas del sector de la industria de la transformación, atendiendo a la similitud de su operación; así tenemos: Alimentos, Bebidas y Tabaco; Industria de la Transformación I; e Industria de la Transformación II.

Al cierre del año 2000 el total de las empresas que cotizan en la Bolsa Mexicana de Valores son 170, pero que actualmente — según Yamashiro (2001: 20) — sólo se encuentran operando, en promedio, 65 de ellas. Así también es importante saber que el IPC ${ }^{15}$ al 31 de diciembre de 2000 se encontraba integrado por 34 empresas $^{16}$, mismas que se encuentran contenidas en esta muestra.

\section{Aplicación del modelo Black y Scholes a las empresas de la muestra}

Al realizar el cálculo del valor de las empresas con la metodología propuesta en el punto II, se obtuvo el valor de las empresas que conforman la muestra para cada uno de los 10 años objeto de estudio (ver anexo No 2). Los datos de las variables financieras se tomaron de los estados financieros publicados en los anuarios financieros de la Bolsa Mexicana de Valores.

El detalle del cálculo del valor de Black y Scholes para cada una de las empresas de la muestra se presenta en el anexo No 3; se muestra sólo el cálculo correspondiente al año 2000 por cuestiones de espacio.

Los valores promedio por sector aplicando el modelo Black y Scholes se calcularon como un promedio simple del valor de las empresas que conforman cada sector; estos valores promedio se presentan a continuación:

15 Índice de Precios y Cotizaciones de la Bolsa Mexicana de Valores.

${ }^{16}$ Dato obtenido de: http:// www.bmv.com.mx, Bolsa Mexicana de Valores al 31.12.2000. 


\section{Cuadro2}

\section{Valor promedio sectorial del modelo Black y Scholes (1991-2000) Empresas que cotizan en la Bolsa Mexicana de Valores (Miles de pesos)}

\begin{tabular}{|c|c|c|c|c|c|c|c|c|c|c|}
\hline Sector & 1991 & 1992 & 1993 & 1994 & 1995 & 1996 & 1997 & 1998 & 1999 & 2000 \\
\hline & & & & & & & & & & \\
\hline ALIMENTOS, BEBIDAS Y TABACO & $7,542,871$ & $7,671,394$ & $8,403,974$ & $12,307,808$ & $10,548,551$ & $9,530,693$ & $11,549,388$ & $11,185,714$ & $13,540,775$ & $14,711,695$ \\
\hline COMERCIO & $6,282,910$ & $7,492,361$ & $9,502,653$ & $10,846,839$ & $7,422,746$ & $7,394,033$ & $9,148,954$ & $8,634,191$ & $9,165,930$ & $10,003,058$ \\
\hline COMUNICACIONES Y TRANSPORTES & $29,431,873$ & $21,155,197$ & $27,860,743$ & $37,688,365$ & $22,887,161$ & $19,890,602$ & $19,202,757$ & $34,881,533$ & $38,701,441$ & $38,393,657$ \\
\hline INDUS TRIA DE LA CONSTRUCCIÓN & $11,801,159$ & $19,173,276$ & $16,049,119$ & $24,605,499$ & $23,494,751$ & $15,902,621$ & $19,157,112$ & $20,244,347$ & $18,625,591$ & $22,546,585$ \\
\hline CONTR OLADORAS & $14,399,522$ & $14,751,645$ & $18,172,666$ & $21,249,271$ & $19,029,176$ & $13,745,440$ & $12,156,154$ & $17,922,447$ & $15,239,108$ & $19,551,671$ \\
\hline $\begin{array}{l}\text { IND. TRANSF.I: METALICOS, MINERA, MINERAL } \\
\end{array}$ & $11,106,671$ & $11,111,623$ & $13,312,791$ & $16,109,170$ & $18,552,951$ & $14,446,594$ & $16,670,126$ & $17,765,951$ & $19,750,265$ & $18,487,872$ \\
\hline IND. TRANSF.II: CELULOSA Y PAPEL Y QUIMICA & $5,784,068$ & $5,538,210$ & $5,079,655$ & $6,507,786$ & $5,553,101$ & $6,010,790$ & $6,169,300$ & $6,056,721$ & $5,803,139$ & $5,550,854$ \\
\hline SERVICIOS FINANCIEROS & n.d & n.d & n.d & n.d & n.d & n.d & $78,106,966$ & $94,090,794$ & $70,252,699$ & $94,359,831$ \\
\hline OTROS SERVICIOS & n.d. & n.d. & 366,022 & 586,678 & 699,361 & 518,522 & 712,215 & 707,840 & $5,269,151$ & $5,599,065$ \\
\hline
\end{tabular}

Los espacios donde aparece n.d. significa que no se tuvo información disponible para realizar el cálculo del valor de las empresas.

A continuación se presenta el precio promedio de mercado para cada uno de los sectores analizados:

Cuadro3

Empresas que cotizan en la Bolsa Mexicana de Valores (Miles de pesos)

\begin{tabular}{|c|c|c|c|c|c|c|c|c|c|c|}
\hline Sector & 1991 & 1992 & 1993 & 1994 & 1995 & 1996 & 1997 & 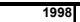 & 1999 & 2000 \\
\hline \begin{tabular}{|l} 
ALIMENTOS, BEBIDAS Y TABACO \\
\end{tabular} & $2,289,276$ & $2,721,288$ & $4,185,760$ & $3,894,291$ & $5,057,564$ & $7,337,672$ & $10,326,734$ & $11,212,536$ & $10,433,937$ & $8,661,975$ \\
\hline COMERCIO & $3,001,242$ & $4,607,744$ & $6,696,503$ & $7,266,213$ & $5,700,205$ & $7,524,033$ & $15,714,239$ & $8,442,143$ & $11,722,906$ & $10,578,399$ \\
\hline COMUNICACIONES Y TRANSPORTES & $24,466,516$ & $26,702,728$ & $28,811,677$ & $22,141,082$ & $34,646,566$ & $19,918,575$ & $27,806,494$ & $24,089,026$ & $58,017,632$ & $48,375,411$ \\
\hline INDUS TRIA DE LA CONSTRUCCIÓN & $8,606,090$ & $6,356,641$ & $11,661,655$ & $9,938,270$ & $11,211,363$ & $12,506,009$ & $15,747,554$ & $8,687,193$ & $17,701,119$ & $11,304,192$ \\
\hline CONIROLADORAS & $2,616,911$ & $3,260,465$ & $5,727,263$ & $6,938,556$ & $9,177,120$ & $11,317,772$ & $14,678,566$ & $9,174,496$ & $13,399,089$ & $7,036,901$ \\
\hline IND. TRANSF.: METALICOS, MINERA, MINERALES NC & $2,185,189$ & $2,409,494$ & $2,813,822$ & $5,015,050$ & $7,359,830$ & $7,409,811$ & $10,953,165$ & $6,170,561$ & $9,341,312$ & $5,122,883$ \\
\hline IND. TRANSF.: CELULOSA Y PAPELY QUIMICA & $2,604,540$ & $3,309,323$ & $3,442,902$ & $6,149,485$ & $7,948,863$ & $11,371,429$ & $14,433,781$ & $10,960,195$ & $13,728,144$ & $8,960,839$ \\
\hline SERVICIOS FINANCIEROS & $5,551,778$ & $16,301,678$ & $14,427,807$ & $11,644,458$ & $10,942,031$ & $15,078,397$ & $20,810,714$ & $11,832,593$ & $26,431,571$ & $25,113,082$ \\
\hline \begin{tabular}{|l|} 
OTROS SERVICIOS \\
\end{tabular} & n.d. & n.d & n.d & n.d & n.d & n.d & 480,992 & 154,823 & 240,575 & $4,034,823$ \\
\hline
\end{tabular}

Si consideramos como patrón de referencia el precio de mercado ${ }^{17}$ de las empresas, se observa que por lo general el valor que arroja el modelo de Black y Scholes es superior al precio de mercado, por lo cual podemos señalar que:

${ }^{17}$ El precio de mercado se obtuvo del Anuario Bursátil de la Bolsa Mexicana de Valores. El mismo se conceptualiza como el precio de la acción multiplicada por el número de acciones en circulación. 
- Se observa una tendencia en el modelo Black y Scholes de arrojar valores mayores y positivos con respecto al valor de mercado, lo cual se puede interpretar como una sobrevaluación del valor de la empresa. Esto se debe básicamente a que el modelo considera como variable para determinar el valor, el valor del activo total, que es muy grande en estas empresas debido a que trabajan con una fuerte inversión en capital de trabajo y activo fijo. Así también, al considerar la volatilidad como variable en este modelo nos permite definir que cuando el riesgo es muy alto (como en el caso de las empresas mexicanas, que operan en un entorno volátil e inestable), los valores que arroja este modelo también son muy altos, pues este modelo privilegia el valor cuando el riesgo es muy elevado. Por otro lado, al considerar en este modelo el apalancamiento, se logran también valores elevados cuando las empresas tienen un apalancamiento alto porque se transfiere el valor del acreedor al accionista.

- Este resultado es consistente con lo encontrado por Milla (1998:126) en la valuación de AHMSA al aplicar este modelo, el cual arrojó un valor por acción de $\$ 39.06$, muy superior al precio de mercado que era $\$ 16.50$, resultado que la investigadora atribuyó al alto potencial de utilidades que tenía AHMSA; sin embargo, sabemos que esta empresa se declaró en suspensión de pagos en 1998.

El valor Black y Scholes en los sectores: en el gráfico de tendencias del valor Black y Scholes en los sectores, se puede apreciar que todos siguen la misma tendencia a través de los años, es decir, presentan el mismo comportamiento de alzas y bajas; sin embargo, se puede observar que el sector Comunicaciones y Transportes sigue una tendencia más acentuada, de alzas y bajas, lo cual tendría que ver con las altas inversiones en activo fijo con las que trabaja este sector y con la alta volatilidad que caracteriza al mismo. Sucede algo similar con el sector de Servicios Financieros, que muestra un elevado valor con este modelo, puesto que trabaja con fuertes inversiones en activos fijos y también se encuentra sujeto a una alta volatilidad. 


\section{Gráfica 3}

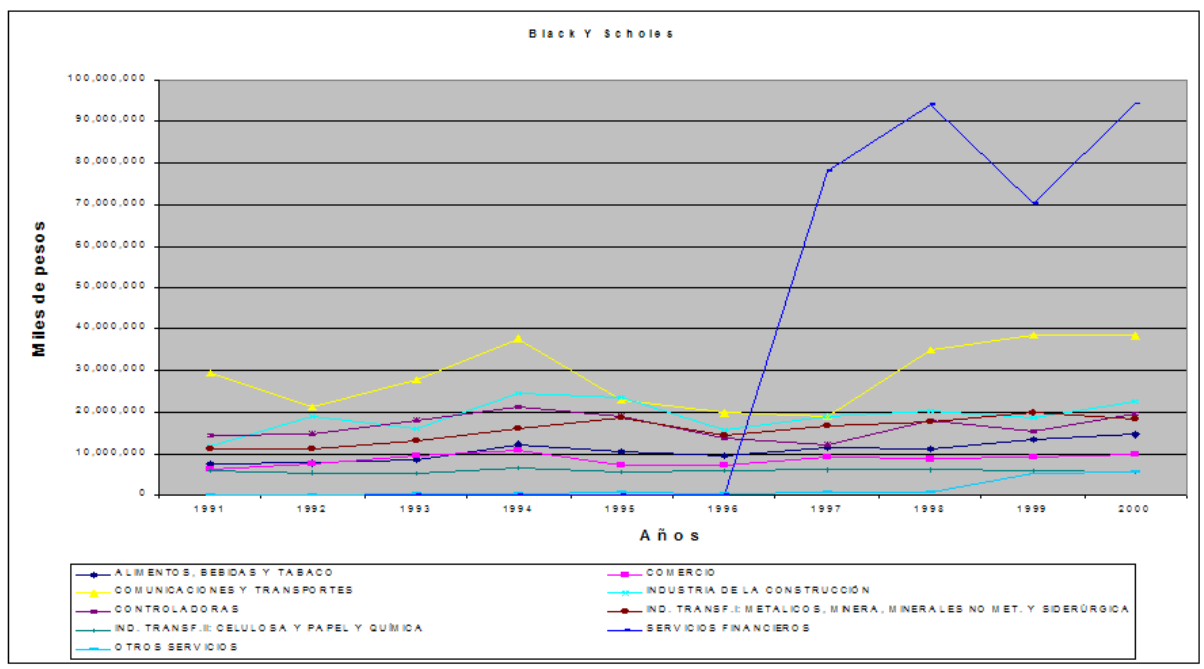

El precio de mercado en los sectores: También observamos en el gráfico de tendencia del precio de mercado que todos los sectores siguen la misma tendencia de alzas y bajas a través del tiempo; sin embargo, nuevamente vemos que el sector Comunicaciones y Transportes sigue una tendencia más acentuada de alzas y bajas, en función de ser un sector de alta volatilidad en el mercado bursátil. 


\section{Gráfica 4}

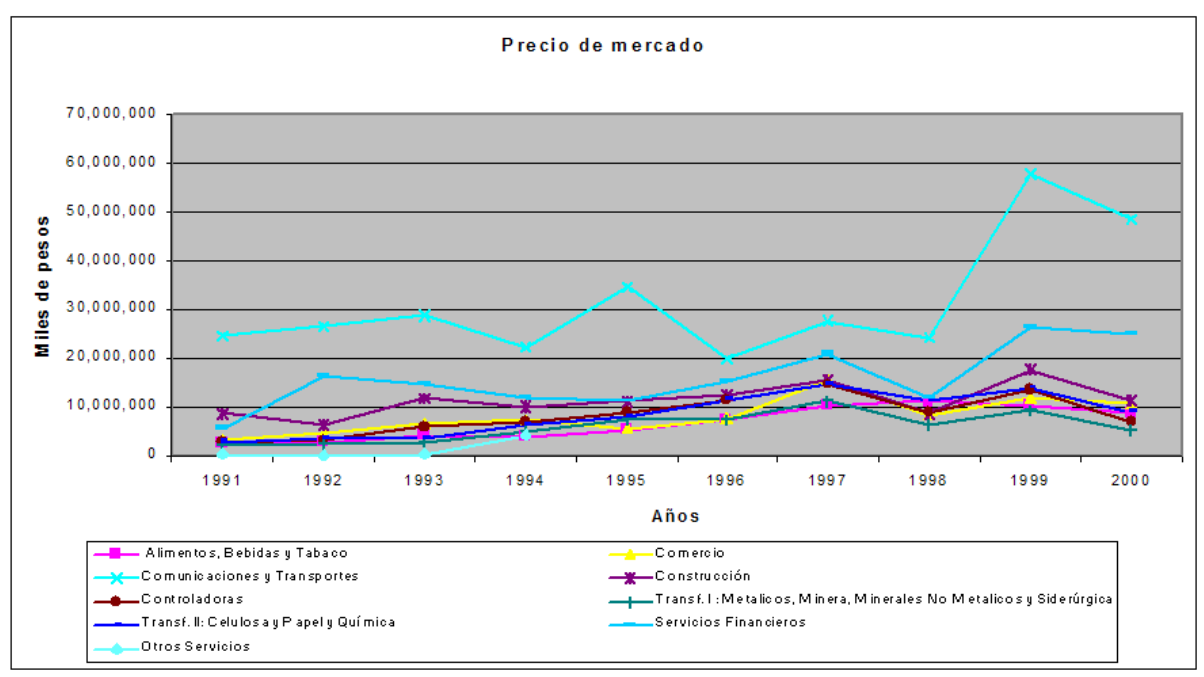

Al realizar un análisis de correlación, de cada uno de los sectores, entre los valores arrojados por el modelo Black y Scholes y el precio del mercado se obtuvo el siguiente resultado

\section{Cuadro 4}

\begin{tabular}{|c|c|}
\hline Sector & $\begin{array}{c}\text { Correlación } \\
\text { Black y } \\
\text { Scholes/ } \\
\text { Mercado } \\
\end{array}$ \\
\hline Alimentos, Bebidas y Tabaco & 0.6774 \\
\hline Comercio & 0.5468 \\
\hline Comunicaciones y Transportes & 0.5099 \\
\hline Construcción & 0.0055 \\
\hline Controladoras & -0.3084 \\
\hline $\begin{array}{l}\text { Transf. I : Metal., Minera, Miner. No Met. y } \\
\text { Siderúrgica }\end{array}$ & 0.7196 \\
\hline Transf. II: Celulosa y Papel y Química & 0.4392 \\
\hline Servicios Financieros & 0.6291 \\
\hline Otros Servicios & 0.7185 \\
\hline
\end{tabular}


En el cuadro anterior observamos que una gran parte de los sectores (Alimentos, Bebidas y Tabaco; Comercio; Comunicaciones y Transportes; Transformación I: Metales, Minera, Minerales No Metálicos y Siderúrgica; Servicios Financieros; Otros Servicios; muestran una correlación superior a 50\%; esto podría estar indicando que el Black y Scholes es un buen modelo para valuar correctamente estos sectores, sin que esto sea contundente.

Así también vemos que los sectores Construcción y Transformación II: Celulosa y Papel y Química muestran una correlación débil, mientras que el sector de Controladoras muestra correlación negativa; ambos aspectos indicarían que este modelo no es adecuado para valuar esos sectores, sin que esto sea contundente.

\subsection{Prueba de hipótesis}

Se enuncia la hipótesis estadística con el fin de realizar la prueba de hipótesis.

$\mathrm{H}_{1}$ : La media de los valores calculados para los sectores con el modelo Black y Scholes es diferente a la media del precio de mercado para los sectores en el período bajo estudio.

También se enuncia la hipótesis nula:

Ho: La media de los valores calculados para los sectores con el modelo Black y Scholes no es diferente a la media del precio de mercado para los sectores en el período bajo estudio.

\section{Resultados de la prueba de hipótesis}

Se aplicó la prueba t de diferencia de medias con un 95\% de confianza; a continuación se muestran los resultados obtenidos: 


\section{Cuadro5}

\begin{tabular}{|l|c|c|l|}
\hline \multicolumn{1}{|c|}{ Sector } & tcalculada & $\begin{array}{c}\text { Valor } \\
\text { crítico de t }\end{array}$ & \multicolumn{1}{|c|}{ Decisión Estadística } \\
\hline $\begin{array}{l}\text { Alimentos, Bebidas y } \\
\text { Tabaco }\end{array}$ & 5.16 & 1.8831 & Se rechaza la hipótesis nula \\
\hline Comercio & 0.462 & 1.8331 & $\begin{array}{l}\text { No se puede rechazar la } \\
\text { hipótesis nula }\end{array}$ \\
\hline $\begin{array}{l}\text { Comunicaciones y } \\
\text { Transportes }\end{array}$ & -0.733 & 1.8331 & $\begin{array}{l}\text { No se puede rechazar } \\
\text { la hipótesis nula. }\end{array}$ \\
\hline Construcción & 4.797 & 1.8331 & Se rechaza la hipótesis nula \\
\hline Controladoras & 4.623 & 1.8331 & Se rechaza la hipótesis nula \\
\hline $\begin{array}{l}\text { Transf. I: Metal., Minera, } \\
\text { Miner. No Met. } \\
\text { y Siderúrgica }\end{array}$ & 13.68 & 1.8331 & Se rechaza la hipótesis nula \\
\hline $\begin{array}{l}\text { Transf. II: } \\
\text { Celulosa, Papel } \\
\text { yQuímica }\end{array}$ & -1.88 & 1.8331 & Se rechaza la hipótesis nula \\
\hline Serivicios Financieros & 7.68 & 2.35 & Se rechaza la hipótesis nula \\
\hline $\begin{array}{l}\text { Otros Servicios } \\
\text { Sing }\end{array}$ & 1.67 & 2.35 & $\begin{array}{l}\text { No se puede rechazar la } \\
\text { hipótesis nula }\end{array}$ \\
\hline
\end{tabular}


Como puede observarse, se rechazaron las hipótesis nulas para seis sectores: Alimentos, Bebidas y Tabaco; Construcción; Controladoras; Transf. I : Metal., Minera, Miner. No Met. y Siderúrgica; Transf. II: Celulosa y Papel y Química; y Servicios Financieros. Lo anterior indica que los valores calculados con el modelo Black y Scholes son diferentes al precio de mercado. Por otra parte, no se pudieron rechazar las hipótesis nulas para tres sectores: Comercio; Comunicaciones y Transportes; y otros servicios; dado que éstos muestran valores de mercado muy elevados y similares a los que arroja el modelo Black y Scholes.

\section{Conclusiones}

El modelo de valuación de opciones aplicado a la valuación de empresas considera que la deuda de la empresa es vista como una opción de compra sobre el capital de la misma; de esta forma, si a la fecha de vencimiento de la deuda el valor de la empresa es mayor que la deuda, los acreedores no ejercen su opción; pero si al vencimiento de la deuda el valor de la empresa es menor que la deuda, los acreedores pueden ejercer su opción de compra y quedarse con la empresa.

La aplicación empírica del modelo de valuación de Black y Scholes muestra una sobreestimación consistente del valor de las empresas que cotizan en la Bolsa Mexicana de Valores por el período de 1991-2000. Asimismo, este modelo tiende a arrojar valores mayores y positivos en comparación con el precio de mercado, lo cual se puede interpretar como una sobrevaluación del valor de la empresa; debido básicamente a que el modelo considera como variable para determinar el valor de la empresa al valor del activo total, que es muy grande en estas empresas, puesto que trabajan con una fuerte inversión en capital de trabajo y activo fijo.

Por otra parte, al considerar en este modelo a la volatilidad como variable para el cálculo del valor nos permite definir que cuando el riesgo es muy alto los valores que arroja también son muy altos porque privilegia el valor cuando el riesgo es muy elevado. Asimismo, al considerar el apalancamiento se logran también valores elevados, ya que se transfiere el valor del acreedor al accionista.

Estos resultados son consistentes con los hallazgos que en 1996 hizo Milla (1998: 126) en la valuación de AHMSA al aplicar este modelo tomando las variables sugeridas por Luehrman, el cual arrojó un valor por acción de $\$ 39.06$, muy superior al precio de mercado que era $\$ 16.50$, resultado que la investigadora atribuyó al 
alto potencial de utilidades que tenía AHMSA; sin embargo, sabemos que esta empresa se declaró en suspensión de pagos en el año 1998, lo cual estaría indicando claramente que este modelo sobrestimó el valor de AHMSA.

Al realizar la prueba de correlación entre los valores de Black y Scholes y el precio de mercado encontramos altas correlaciones en seis de los nueve sectores analizados, lo que podría estar indicando que es un modelo adecuado para calcular el valor de las empresas en estos sectores, sin que esto sea contundente.

También, de acuerdo con la prueba $t$ de diferencia de medias, sólo se pudieron rechazar seis hipótesis nulas, sin tener suficiente fundamento estadístico para rechazar las hipótesis en tres sectores; esto indicaría que son sectores que alcanzaron un precio de mercado muy elevado y similar al valor que arroja el modelo Black y Scholes.

La aplicación de este modelo al conjunto de empresas mexicanas por el período estudiado permite definir que hay que tener cuidado en la aplicación del mismo para efectos de valuación, considerando entre otros aspectos las características propias de la empresa como son la inversión fija y el apalancamiento. Además, hay que tomar en cuenta que se ha demostrado que es un modelo que privilegia el valor cuando el riesgo es elevado.

\section{Bibliografía}

Abreu, Martín (1999), Valuación de Rendimientos Esperados en opciones bursátiles de compra en un mercado agropecuario en México, México: UNAM-FCA, Tesis de grado de doctorado en Administración.

Adam, Juan A. (1996), “Aplicando el Modelo de Black y Scholes, el método de valor en libros, de valor de mercado, el del múltiplo: precio-utilidad, y el de Goodwill", México: Contaduría y Administración, No. 182, julio-septiembre, pp. 33-42.

Alzugaray, Angélica (2000), "The Black and Scholes Method for Option Valuation Applied to the Evaluation of Investment Projects", México: The Anáhuac Journal, Vol.2, No. 2, pp. 112-121. 
Bolsa Mexicana de Valores (2001), Anuario bursátil de la Bolsa Mexicana de Valores, México: Bolsa Mexicana de Valores.

Bolsa Mexicana de Valores (2001), Anuario financiero de la Bolsa Mexicana de Valores, México: Bolsa Mexicana de Valores.

Baskshi, Gurdip y Chen Zhiwu (1997), "An Alternative Valuation Model for Contingent Claims", EUA : Journal of Financial Economics, Vol. 44, No. 1, pp. 123-165.

Bellalah, Mondher y Jacquillat Bertrand (1995), "Option Valuation with information cost: Theory and tests", EUA: The Financial Review, Vol. 30, No. 3, agosto, pp. 617-636.

Black, Fischer y Scholes Myron (1973), "The pricing of options and corporate liabilities", EUA : Journal of Political Economy, Vol. 81, No. 3, mayo/junio, pp. 635-676.

Coller, Maribeth y Julia Higgs (1997), "Firm valuation and accouting for employee stock options", EUA: Financial Analysts Journal, Vol. 53, No. 1, enero/ febrero, pp. 26-34.

Copeland, Tom y Philip Keenan (1998), "Making real options real”, EUA: The McKinsey Quarterly, No. 3, pp. 128-141.

Damodaran, Aswath (1994), Damodaran on Valuation, Security Analysis for Investment and Corporate Finance, EUA: John Wiley \& Sons, Inc.

(2000), Value Creation and Enhancement: Back to the Future, Nueva York : Stern School of Business.

Díaz, Carmen (1998), Futuros y opciones sobre futuros financieros, México: Prentice Hall.

Díaz, Jaime y Fausto Hernández (1998), Futuros y opciones financieras, segunda edición, México: Limusa. 
Galitz, Lawrence (1994a), Ingeniería financiera I, España: Folio.

Koller, Timothy (1994), “What is value-based management?”, EUA: The Mc. Kinsey Quartely No. 3, pp. 87-101.

Leslie, Keith y Max Michaels (1997), "The real Power of Real Options", EUA: The McKinsey Quartely, No. 3, pp. 4-22.

López, Francisco (2001), "Trampas en la valoración de negocios”, España: Harvard Deusto Business Review, marzo-abril, pp. 1-10.

Luehrman, Timothy (1998a), "Investment Opportunities as Real Options: Getting Started on the Numbers", EUA: Harvard Business Review, julio-agosto, pp. 89-99.

(1998b), "Strategy as a Portfolio of Real Options", EUA: Harvard Business Review, septiembre-octubre.

Martínez, Eduardo (1993), Futuros y opciones en la gestión de carteras, España: Mc Graw Hill.

Mascareñas Pérez Iñigo, Juan (2000b), "Métodos de Valoración de empresas de la nueva economía”, España: Contabilidad y finanzas, Vol. 88, mayo, pp. 6-12.

Merton, Robert y Scott Mason (1985), Recent Advances in Corporate Finance: The Role of Contingent Claims Analysis in Corporate Finance, EUA: Irwin.

Merton, Robert (1997), Cases in Financial Engineering. Applied Studies of Financial Innovation, EUA: Prentice Hall, Englewood Cliffs.

Milla, Sindy (1998), El análisis fundamental y la valuación de acciones en México, México: UNAM, Facultad de Contaduría y Administración, Tesis de grado de maestría en Finanzas. 
Rappaport, Alfred (1986), Creating Shareholder value, Nueva York: The Free Press.

Rodríguez de Castro, Juan (1998), Introducción al análisis de Productos Financieros Derivados, México: Bolsa Mexicana de Valores, y Limusa.

Simón, Nadima (1994), Evaluación organizacional: El caso de Altos Hornos de México, S.A. después de la privatización, México: UNAM-FCA, Tesis de grado de doctorado en Administración.

Simons, Katerina (1997), "Model Error", Inglaterra: New England Economic Review, noviembre-diciembre, pp. 17-29.

Stewart, Myers (1996), "Fischer Black's contributions to corporate finance", Tampa: Financial Management, Vol. 25, No. 4, pp- 95-103.

Taggart, James, Peter Kontes y Michael Mankins (1994), The Value Imperative: Managing for Superior Shareholder Returns, Nueva York: The Free Press.

Thierauf, Robert y Richard Grosse (1987), Toma de decisiones por medio de investigación de operaciones, México: Limusa.

Van Horne, James (1993), Administración financiera, México: Prentice Hall.

Yamashiro, Celina (2001), "Las empresas en la Bolsa Mexicana de Valores", México: El Financiero, Sección de Negocios, 25 de junio, p. 20. 


\section{Anexo 1}

Empresas que conforman la muestra

\begin{tabular}{|l|l|}
\hline No & Sector 1: Alimentos, Bebidas y Tabaco \\
\hline 1 & Grupo Bimbo, S.A. de C.V. \\
\hline 2 & Grupo Continental, S.A. de C.V. \\
\hline 3 & Fomento Económico Mexicano, S.A. de C.V. \\
\hline 4 & Grupo Industrial Maseca, S.A. de C.V. \\
\hline 5 & Grupo Modelo, S.A. de C.V. \\
\hline 6 & Savia, S.A. de C.V. \\
\hline 7 & Industrias Bachoco, S.A. de C.V. \\
\hline 8 & Grupo de Embotelladoras Unidas, S.A. de C.V. \\
\hline 9 & Grupo La Moderna, S.A. de C.V. \\
\hline 10 & Embotelladoras Argos, S.A. de C.V. \\
\hline 11 & Grupo Herdez, S.A. de C.V. \\
\hline 12 & Maizoro, S.A. de C.V. \\
\hline 13 & Coca Cola Femsa, S.A. de C.V. \\
\hline 14 & Gruma, S.A.de C.V. \\
\hline & \\
\hline & \\
\hline & Sector 2: Comercio \\
\hline 15 & Controladora Comercial Mexicana, S.A. de C.V. \\
\hline 16 & Organización Soriana, S.A. de C.V. \\
\hline 17 & Grupo Elektra, S.A. de C.V. \\
\hline 18 & Wall Mart México, S.A. de C.V. \\
\hline 18 & Grupo Casa Saba, S.A. de C.V. \\
\hline 20 & Grupo Dataflux, S.A. de C.V. \\
\hline 21 & Edoardos Martín, S.A. de C.V. \\
\hline 22 & Corporativo Fragua, S.A. de C.V. \\
\hline 23 & Grupo Corvi, S.A. de C.V. \\
\hline 24 & Grupo Gigante, S.A. de C.V. \\
\hline 25 & Grupo Palacio de Hierro, S.A. de C.V. \\
\hline 26 & Alsea, S.A. de C.V. \\
\hline 28 & Nadro, S.A. de C.V. \\
\hline & Puerto de Liverpool, S.A. de C.V. \\
\hline 29 & Grupo Prove-Quim. S.A. de C.V. \\
\hline
\end{tabular}




\section{Empresas que conforman la muestra}

\begin{tabular}{|l|l|}
\hline & Sector 3: Comunicaciones \\
\hline 30 & Carso Global Telecom, S.A. de C.V. \\
\hline 31 & Grupo Televisa, S.A. \\
\hline 32 & Teléfonos de México, S.A. de C.V. \\
\hline 33 & TV Azteca, S.A. de C.V. \\
\hline 34 & Transportación Marítima Mexicana, S.A. de C.V. \\
\hline 35 & Cintra, S.A. de C.V. \\
\hline 36 & Grupo Radio Centro, S.A. de C.V. \\
\hline 37 & Biper, S.A. de C.V. \\
\hline 38 & Grupo Iusacell, S.A. de C.V. \\
\hline & \\
\hline 39 & Sector 4: Construcción \\
\hline 40 & Apasco, S.A. de C.V. \\
\hline 41 & Cemex, S.A. de C.V. \\
\hline 42 & Consorcio Ara, S.A. de C.V. \\
\hline 43 & Corporación Geo, S.A. de C.V. \\
\hline 44 & Empresas Ica Sociedad Controladora, S.A. de C.V. \\
\hline & \\
\hline & Sector 5: Controladoras \\
\hline 45 & Corporación Interamericana de Entretenimiento, S.A. de C.V. \\
\hline 46 & Alfa, S.A. de C.V. \\
\hline 47 & Desc, S.A. de C.V. \\
\hline 48 & Grupo Carso, S.A. de C.V. \\
\hline 49 & Grupo Industrial Saltillo, S.A. de C.V. \\
\hline 50 & Grupo Sanbors, S.A. de C.V. \\
\hline 51 & San Luis Corporación, S.A. de C.V. \\
\hline 52 & Cydsa, S.A. de C.V. \\
\hline 54 & G. Acción, S.A. de C.V. \\
\hline & Grupo Industrial Camesa, S.A. de C.V. \\
\hline & \\
\hline & \\
\hline & \\
\hline & \\
\hline & \\
\hline & \\
\hline & \\
\hline & \\
\hline & \\
\hline & \\
\hline & \\
\hline & \\
\hline & \\
\hline & \\
\hline &
\end{tabular}




\section{Empresas que conforman la muestra}

\begin{tabular}{|l|l|}
\hline & $\begin{array}{l}\text { Sector 6: Ind. Transformación I :Metálicos, Minera, } \\
\text { Minerales no Metálicos y Siderúrgica }\end{array}$ \\
\hline 55 & Metálicos Industrias CH, S.A. de C.V. \\
\hline 56 & Minera Grupo México, S.A. de C.V. \\
\hline 57 & Minera Industrias Peñoles, S.A. de C.V. \\
\hline 58 & Minerales no M Vitro, S.A. de C.V. \\
\hline 59 & Siderúrgica Tubos de Acero de México, S.A. de C.V. \\
\hline 60 & Siderúrgica Hylsamex, S.A. de C.V. \\
\hline & \\
\hline & $\begin{array}{l}\text { Sector 7: Ind. Transformación II :Celulosa y Papel y } \\
\text { Química }\end{array}$ \\
\hline 61 & Celulosa/Papel Kimberly Clark de México, S.A.de C.V. \\
\hline 62 & Celulosa/Papel Empaques Ponderosa, S.A. de C.V. \\
\hline 63 & Química Tekchem S.A. de C.V. \\
\hline 64 & Química Mexichem S.A. de C.V. \\
\hline & \\
\hline & Sector 8: Servicios Financieros \\
\hline 65 & Serv. Financieros Grupo Financiero, Banamex Accival, \\
\hline & S.A. de C.V, \\
\hline 66 & Serv. Financieros Grupo Financiero Bital, S.A. de C.V. \\
\hline 67 & Serv. Financieros Grupo Financiero BBVA Bancomer, \\
\hline & S.A. de C.V. \\
\hline 68 & Serv. Financieros Grupo Financiero Inbursa, S.A. de C.V. \\
\hline 69 & Serv. Financieros Grupo Financiero Banorte, S.A. de C.V. \\
\hline & \\
\hline 70 & Sector 9: Otros Servicios \\
\hline 71 & Médica Sur, S.A. de C.V. \\
\hline & \\
\hline
\end{tabular}

Fuente: BMV (2001), Anuario bursátil 2000, México: Bolsa Mexicana de Valores 
Anexo 3

Valores calculados aplicando el modelo Black y Scholes

miles de pesos

\begin{tabular}{|c|c|c|c|c|c|c|c|c|c|c|c|c|c|c|c|}
\hline \multicolumn{16}{|c|}{ (miles de pesos) } \\
\hline & Activo $^{1}$ & Pasivo $^{2}$ & Costo del ${ }^{3}$ & \begin{tabular}{|l|l|} 
Pasivoa $^{4}$ & $\mathrm{~T}$ \\
\end{tabular} & Tasa real $^{5}$ & Volatilidad ${ }^{6}$ & Tpo ${ }^{7}$ & $e^{8}$ & $\mathbf{s} / \mathbf{X}^{9}$ & Lg. N. ${ }^{10}$ & $\mathrm{~d} 1^{11}$ & Normal $^{12}$ & $\mathrm{~d} 2^{13}$ & Normal $^{12}$ & $\mathrm{C}^{14}$ \\
\hline BYS 2000 & 2000 & 2000 & Pasivo & Futuro & & \begin{tabular}{|l|} 
Stan dard \\
\end{tabular} & Vcmto. & 2.71828 & & $\mathrm{~s} / \mathrm{X}$ & & & & & \\
\hline No|Empresa & $\mathrm{s}$ & & & $\mathrm{x}$ & & & & & & & & & & & \\
\hline 1 BIMBO & $24,898,333$ & $4,377,298$ & 0.0581 & $5, \hat{5,185,393}$ & $5.30 \%$ & $37.58 \%$ & & 2.71828 & 4.80162891 & 1.57 & 1.940 & 0.967800 & 1.2889 & 0.884900 & $20,182,586$ \\
\hline $\begin{array}{ll}2 & \text { CONTINENTAL GPO. } \\
\end{array}$ & $7,910,324$ & 168,692 & 0.0111 & 174,367 & $5.30 \%$ & $41.81 \%$ & & 2.71828 & 45.3659604 & 3.81 & 4.236 & 1.000000 & 3.5118 & 0.999760 & $7,761,625$ \\
\hline \begin{tabular}{l|l} 
FEMSA \\
3
\end{tabular} & $45,684,849$ & $9,061,101$ & 0.0746 & $11,242,638$ & $5.30 \%$ & $46.22 \%$ & & 2.71828 & 4.0635347 & 1.40 & 1.881 & 0.961600 & 1.0809 & 0.834000 & $\begin{array}{r}35,932,549 \\
\end{array}$ \\
\hline $\begin{array}{ll}\text { GMASECA } \\
\end{array}$ & $22,070,000$ & $7,020,687$ & 0.0739 & $8,695,559$ & $5.30 \%$ & $34.25 \%$ & & 2.71828 & 2.5380771 & 0.93 & 1.266 & 0.877000 & 0.6731 & 0.712300 & $14,072,061$ \\
\hline \begin{tabular}{|l|l}
5 & GMODELO \\
\end{tabular} & $45,810,077$ & $\mid \frac{1,20,000}{267,000}$ & 0.0080 & \begin{tabular}{r|}
$0,30,343$ \\
273,443 \\
\end{tabular} & $5.30 \%$ & $40.23 \%$ & & 2.71828 & 167.530476 & 5.12 & 5.523 & \begin{tabular}{|l|l|}
3.000000 \\
\end{tabular} & 4.8261 & 1.000000 & $45,576,831$ \\
\hline \begin{tabular}{|l|l|}
6 SAVIA \\
\end{tabular} & $63,557,539$ & $12,073,265$ & 0.0732 & $14,923,559$ & $5.30 \%$ & $14.76 \%$ & 3 & 2.71828 & 4.25887285 & 1.45 & 1.641 & 0.937000 & 1.3850 & 0.899700 & $48,100,465$ \\
\hline \begin{tabular}{l|l|}
7 & BACHOCO \\
\end{tabular} & $9,736,651$ & 768,386 & 0.1143 & $1,063,047$ & $5.30 \%$ & $41.53 \%$ & & 2.71828 & 9.15919117 & 2.21 & 2.632 & 0.993200 & 1.9131 & 0.959900 & $8,800,028$ \\
\hline 8 GEM UNIDAS & $2,012,539$ & 114,315 & 0.1370 & 168,047 & $5.30 \%$ & $36.50 \%$ & 3 & 2.71828 & 11.9760785 & 2.48 & 2.842 & 0.996000 & 2.2096 & 0.978300 & $1,864,256$ \\
\hline \begin{tabular}{l|l}
9 & GMODERNA \\
\end{tabular} & $2,559,071$ & 270,006 & 0.1264 & 385,865 & $5.30 \%$ & $36.50 \%$ & 3 & 2.71828 & 6.63203937 & 1.89 & 2.251 & 0.981200 & 1.6186 & 0.925100 & $2,206,472$ \\
\hline \begin{tabular}{l|l}
10 & ARGOS \\
\end{tabular} & $4,028,703$ & 628,885 & 0.1505 & 957,817 & $5.30 \%$ & $34.08 \%$ & 3 & 2.71828 & 4.20613031 & 1.44 & 1.770 & 0.941800 & 1.1795 & 0.836500 & $3,110,800$ \\
\hline \begin{tabular}{l|l}
11 HERDEZ, S.A. \\
\end{tabular} & $3,748,385$ & $1,079,992$ & 0.0935 & $1,412,322$ & $5.30 \%$ & $59.63 \%$ & & 2.71828 & 2.65405808 & 0.98 & 1.668 & \begin{tabular}{ll|}
3 & 0.937000 \\
\end{tabular} & 0.6356 & 0.691500 & $2,679,183$ \\
\hline $\begin{array}{l}12 \text { MAIZORO } \\
\text {.A. }\end{array}$ & $\begin{array}{r}3,46,000 \\
39,829 \\
\end{array}$ & | & $\begin{array}{ll}0.0353 \\
0.1093 \\
\end{array}$ & $\mid \frac{\mid, 412,322}{132,547}$ & $5.30 \%$ & $36.50 \%$ & & $\frac{2.17000}{2.71828}$ & 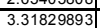 & $\frac{1.90}{1.20}$ & $\frac{1.500}{1.558}$ & \begin{tabular}{|l|l|}
3 & 0.919200 \\
\end{tabular} & 0.0261 & 年 & $\frac{2,019,103}{316,171}$ \\
\hline \begin{tabular}{l|l}
13 \\
KOF COCA COLA
\end{tabular} & $12,910,753$ & $4,046,765$ & 0.0548 & $4,748,848$ & $5.30 \%$ & $36.50 \%$ & 3 & 2.71828 & 2.71871239 & 1.00 & 1.359 & 0.899700 & 0.7268 & 0.738900 & $8,622,705$ \\
\hline 14 GMASECA & $6,832,941$ & 22,537 & 0.7012 & 110,963 & $5.30 \%$ & $36.50 \%$ & & 2.71828 & 61.5783805 & 4.12 & 4.479 & 0.999950 & 3.8469 & 0.999420 & $6,738,003$ \\
\hline $15 \mid$ KIMBERLY CLARK & $21,005,658$ & $4,065,944$ & 0.0688 & $4,964,641$ & $5.30 \%$ & $38.55 \%$ & & 2.71828 & 4.23105285 & 1.44 & 1.824 & 0.957300 & 1.1567 & 0.853100 & $16,495,990$ \\
\hline \begin{tabular}{l|l}
16 & EMPAQUES POND \\
\end{tabular} & $4,072,681$ & 723,572 & 0.0960 & 952,491 & $5.30 \%$ & $28.29 \%$ & & 2.71828 & 4.27581914 & 1.45 & 1.732 & 0.944100 & 1.2420 & 0.864300 & $3,142,799$ \\
\hline 17 COM. MEXICANA & $20,473,123$ & $1,749,269$ & 0.0884 & $2,255,511$ & $5.30 \%$ & $46.28 \%$ & & 2.71828 & 9.07693206 & 2.21 & 2.686 & 0.994800 & 1.8844 & 0.960800 & $18,518,138$ \\
\hline \begin{tabular}{|l|l|}
18 SORIANA \\
\end{tabular} & $17,559,646$ & 412,186 & 0.0010 & 413,379 & $5.30 \%$ & $44.31 \%$ & & 2.71828 & 42.4783635 & 3.75 & 4.203 & 1.000000 & 3.4350 & 0.999700 & $17,207,142$ \\
\hline \begin{tabular}{l|l|l|l|}
19 & ELEKTRA \\
\end{tabular} & $14,334,858$ & $4,374,679$ & 0.0986 & $5,801,273$ & $5.30 \%$ & $57.74 \%$ & & 2.71828 & 2.4709851 & 0.90 & 1.564 & 0.922200 & 0.5636 & 1.000000 & $8,271,141$ \\
\hline 20 WALL MART MEX & $50,262,308$ & & 0.0000 & & $5.30 \%$ & $50.14 \%$ & & 2.71828 & 50262308.4 & 17.73 & 18.269 & 1.000000 & 17.4004 & 1.000000 & $50,262,308$ \\
\hline 21 SABA CASA GPO. & $6,705,738$ & 688,346 & 0.0363 & 766,020 & $5.30 \%$ & $59.25 \%$ & 3 & 2.71828 & 8.75400408 & 2.17 & 2.855 & 0.997400 & 1.8289 & 0.961600 & $6,059,982$ \\
\hline \begin{tabular}{|l|l|}
22 & DATAFLUX \\
\end{tabular} & $1,044,147$ & 54,756 & 0.0316 & 60,111 & $5.30 \%$ & $82.70 \%$ & & 2.71828 & 17.3703408 & 2.85 & 4.040 & 0.999970 & 2.6073 & 0.994800 & 993,108 \\
\hline 23 EDOARDO & 637,559 & 31,878 & 0.1769 & 51,969 & $5.30 \%$ & $59.25 \%$ & & 2.71828 & 12.2680671 & 2.51 & 3.193 & \begin{tabular}{l|l|}
3 & 0.998500 \\
\end{tabular} & 2.1663 & 0.973200 & 593,461 \\
\hline \begin{tabular}{l|l}
24 & FRAGUA CORP.
\end{tabular} & $1,766,437$ & 141,315 & 0.0826 & 179,324 & $5.30 \%$ & $59.25 \%$ & & 2.71828 & 9.8505097 & 2.29 & 2.973 & 0.997800 & 1.9469 & 0.965600 & $1,614,850$ \\
\hline 25 GCORVI & $3,923,679$ & 454,833 & 0.1404 & \begin{tabular}{|l|}
674,489 \\
\end{tabular} & $5.30 \%$ & $50.08 \%$ & 3 & 2.71828 & 5.81726358 & 1.76 & 2.296 & 0.982100 & 1.4286 & 0.892500 & $3,339,957$ \\
\hline 26 GIGANTE & $17,709,601$ & 678,313 & 0.2574 & $1,348,388$ & $5.30 \%$ & $40.68 \%$ & & 2.71828 & 13.1339078 & 2.58 & 2.982 & 0.996200 & 2.2778 & 0.975000 & $16,520,889$ \\
\hline \begin{tabular}{l|l}
27 & GPALACIO HIERRO \\
\end{tabular} & $8,579,886$ & $1,591,317$ & 0.1156 & $2,209,179$ & $5.30 \%$ & $93.09 \%$ & 3 & 2.71828 & 3.88374433 & 1.36 & 2.816 & 0.996000 & 1.2033 & 0.850800 & $6,942,300$ \\
\hline \begin{tabular}{|l|l|}
28 & ALSEA \\
\end{tabular} & $1,466,974$ & 289,859 & 0.0911 & 376,565 & $5.30 \%$ & $59.25 \%$ & & 2.71828 & 3.89567447 & 1.36 & 2.045 & 0.971900 & 1.0192 & 0.813300 & $1,164,513$ \\
\hline 29 NADRO & $4,180,319$ & 50,562 & 0.0664 & 61,315 & $5.30 \%$ & $59.25 \%$ & & 2.71828 & 68.17766 & 4.22 & 4.908 & 1.000000 & 3.8815 & 0.999920 & $4,128,022$ \\
\hline \begin{tabular}{l|l|l|l|l|}
30 & LIVERPOOL \\
\end{tabular} & $16,584,896$ & $2,639,096$ & 0.0571 & $3,117,342$ & $5.30 \%$ & $83.82 \%$ & & 2.71828 & 5.32020416 & 1.67 & 2.884 & & 1.4326 & 0.911500 & $14,118,022$ \\
\hline 31 GPROVE QUIM & 388,683 & $\mid$ & 0.1902 & | & $5.30 \%$ & $59.25 \%$ & & 2.71828 & 4.10464246 & 1.41 & 2.098 & \begin{tabular}{|l|l|}
3 & 0.967800 \\
\end{tabular} & 1.0715 & 0.793900 & 312,042 \\
\hline \begin{tabular}{l|l|l|}
32 & TELECOM CARSO \\
\end{tabular} & $246,341,337$ & $93,338,113$ & 0.0715 & \begin{tabular}{|l|}
$114,838,995$ \\
\end{tabular} & $5.30 \%$ & $55.75 \%$ & & 2.71828 & 2.14510181 & 0.76 & 1.388 & \begin{tabular}{|l|l|}
3 & 0.899700 \\
\end{tabular} & 0.4228 & 0.625500 & $160,361,038$ \\
\hline \begin{tabular}{l|l}
3 & TELEVISA
\end{tabular} & $\frac{240,04,03,833}{45,498}$ & $\frac{0,030,1957}{11,344,957}$ & 0.0811 & \begin{tabular}{|l}
$14,336,662$ \\
1400
\end{tabular} & $5.30 \%$ & $58.97 \%$ & & $\frac{2.71828}{2.71828}$ & 3.17360008 & 1.15 & 1.835 & & 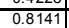 & 0.754900 & $\begin{array}{l}34,324,269 \\
3406\end{array}$ \\
\hline 34 TELMEX & $158,650,234$ & $75,250,656$ & 0.0873 & $96,717,434$ & $5.30 \%$ & $58.11 \%$ & & 2.71828 & 1.64034784 & 0.49 & 1.160 & & 0.1539 & & $92,374,914$ \\
\hline \begin{tabular}{l|l}
35 & TVAZTECA \\
\end{tabular} & $18,826,979$ & $6,076,557$ & 0.0980 & $\begin{array}{l}8,044,003 \\
\end{array}$ & $5.30 \%$ & $66.15 \%$ & 3 & 2.71828 & 2.34049879 & 0.85 & 1.666 & \begin{tabular}{|l|l|}
5.935700 \\
5
\end{tabular} & 0.5200 & 0.644300 & $13,195,536$ \\
\hline \begin{tabular}{|l|l|}
36 & TMM \\
\end{tabular} & $26,004,716$ & \begin{tabular}{|l|}
$12,292,493$ \\
\end{tabular} & 0.0301 & $13,434,911$ & $5.30 \%$ & $75.71 \%$ & & 2.71828 & 1.93560762 & 0.66 & 1.679 & 0.948400 & 0.3679 & 0.625500 & $17,494,686$ \\
\hline \begin{tabular}{|l|l|}
37 & CINTRA \\
\end{tabular} & $20,661,867$ & $5,606,072$ & 0.0742 & $6,948,613$ & $5.30 \%$ & $67.26 \%$ & & 2.71828 & 2.9735239 & 1.09 & 1.927 & & 0.7624 & 0.742200 & $15,551,974$ \\
\hline \begin{tabular}{|l|l}
38 & RADIO CENTROO \\
\end{tabular} & 1,739,902 & $\mid \frac{3,000,794}{338,}$ & 0.0326 & 373,037 & $5.30 \%$ & $86.40 \%$ & & 2.71828 & 4.6641544 & 1.54 & 2.819 & 0.997200 & 1.3222 & 0.898000 & $1,449,287$ \\
\hline 39 MOVIL ACCES & 552,243 & 4,802 & 0.1505 & 7,313 & $5.30 \%$ & $68.37 \%$ & & 2.71828 & 75.5135431 & 4.32 & 5.184 & 1.000000 & 4.0003 & 0.999920 & 546,005 \\
\hline \begin{tabular}{l|l} 
IU IUSACELL \\
\end{tabular} & $15,957,887$ & $7,7,756,412$ & $\begin{array}{ll}0.0876 \\
0.0876\end{array}$ & $\mid$ & $5.30 \%$ & $78.67 \%$ & & $\frac{2.71828}{2.718}$ & $\frac{1.59915191}{1.5191}$ & $\begin{array}{ll}\mid .057 \\
0.47\end{array}$ & $\frac{1.047}{1.557}$ & 0.923600 & $\begin{array}{ll}4.1942 \\
0.1942\end{array}$ & 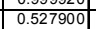 & $10,245,206$ \\
\hline \begin{tabular}{|l|l|l|l|l}
41 APASCO \\
\end{tabular} & $15,509,111$ & $1,768,656$ & 0.0680 & $2,154,363$ & $5.30 \%$ & $35.78 \%$ & & 2.71828 & 7.19893251 & 1.97 & 2.325 & 0.986800 & 1.7052 & 0.945200 & $13,567,431$ \\
\hline \begin{tabular}{l|l}
42 & CEMENTOS CHIHUAHUA \\
\end{tabular} & $5,412,758$ & $\begin{array}{l}1,671,020 \\
\end{array}$ & 0.0423 & $1,892,402$ & $5.30 \%$ & $33.14 \%$ & & 2.71828 & 2.86025867 & 1.05 & 1.375 & 0.904900 & 0.8006 & 0.770400 & $3,654,416$ \\
\hline $\begin{array}{lll}43 & \text { CEMEX, S.A. } \\
\end{array}$ & $153,309,832$ & $56,329,445$ & 0.0524 & $65,658,164$ & $5.30 \%$ & $35.74 \%$ & & 2.71828 & 2.33496981 & 0.85 & 1.199 & 0.868600 & 0.5796 & 0.691500 & $94,436,646$ \\
\hline $\begin{array}{lll}44 \text { ARA } \\
\end{array}$ & & & & 767,000 & 5.30 & & & & & 1.6 & 2.042 & & 1.3597 & & $3,340,730$ \\
\hline 45 GEO & $5,873,363$ & $2,284,692$ & 0.0542 & $2,676,509$ & $5.30 \%$ & $61.02 \%$ & & 2.71828 & 2.1944115 & 0.79 & 1.503 & \begin{tabular}{|l|l|}
3 & 0.922200 \\
\end{tabular} & 0.4465 & 0.640600 & $3,953,892$ \\
\hline \begin{tabular}{l|l}
46 & ICA SOC. CONTROL. \\
\end{tabular} & $24,291,784$ & $7,555,208$ & 0.1128 & $10,411,200$ & $5.30 \%$ & $55.91 \%$ & & 2.71828 & 2.33323579 & 0.85 & 1.475 & 0.904900 & 0.5068 & 0.636800 & $16,326,395$ \\
\hline \begin{tabular}{l|l}
47 & CORP.INTERAMER. \\
\end{tabular} & $\frac{9,942,000}{9}$ & $2,863,491$ & 0.0754 & $3,560,848$ & $5.30 \%$ & $43.86 \%$ & & 2.71828 & 2.79203177 & 1.03 & 1.474 & 0.913100 & 0.7146 & 0.725700 & $6,873,806$ \\
\hline \begin{tabular}{|l|l|}
48 & ALFA \\
\end{tabular} & $73,623,146$ & $32,917,581$ & 0.0688 & $40,189,802$ & $5.30 \%$ & $53.48 \%$ & & 2.71828 & 1.83188625 & 0.61 & 1.193 & & 0.2671 & & $44,149,315$ \\
\hline \begin{tabular}{l|l|l|}
49 & DESC \\
\end{tabular} & $32,325,475$ & $12,251,666$ & 0.0618 & $14,665,844$ & 5.30 & $49.92 \%$ & & 2.71828 & 2.2041333 & 0.79 & 1.323 & & 0.4585 & 0.644300 & $20,732,164$ \\
\hline 50 GCARSO & $85,776,000$ & $28,639,476$ & 0.1025 & $38,381,196$ & $5.30 \%$ & $52.14 \%$ & & 2.71828 & 2.23484436 & 0.80 & 1.371 & 0.888800 & 0.4679 & 0.625500 & $55,759,449$ \\
\hline \begin{tabular}{|l|l|}
51 GIND SALTILLO \\
\end{tabular} & $7,744,216$ & & & $2,262,832$ & 5.30 & $43.59 \%$ & & 2.71828 & 3.42235519 & 1.23 & 1.674 & 0.947400 & 0.9193 & 0.807800 & $5,777,664$ \\
\hline $\begin{array}{ll}52 & \text { GSANBORNS } \\
\end{array}$ & $39,599,000$ & $10,163,051$ & 0.1461 & $15,300,191$ & $5.30 \%$ & $46.77 \%$ & & 2.71828 & 2.58813754 & 0.95 & 1.438 & 0.892500 & 0.6280 & 0.666400 & $26,644,915$ \\
\hline \begin{tabular}{l|l}
53 & SAN LUIS CORP. \\
\end{tabular} & $8,479,085$ & $5,045,451$ & 0.0785 & $6,330,061$ & $5.30 \%$ & $46.72 \%$ & & 2.71828 & 1.33949506 & 0.29 & 0.779 & 0.779400 & -0.0305 & 0.012000 & $6,543,805$ \\
\hline $\begin{array}{ll}54 & \text { CYDSA } \\
\end{array}$ & 12,344,463 & $5,628,401$ & 0.0949 & $7,387,619$ & $5.30 \%$ & $45.88 \%$ & & 2.71828 & 1.67096644 & 0.51 & 0.988 & $\begin{array}{ll}3 & 0.802300 \\
\end{array}$ & 0.1935 & 0.523900 & $6,602,548$ \\
\hline 55 GACCION & $3,566,944$ & $2,123,960$ & 0.0610 & $2,536,774$ & $5.30 \%$ & $49.92 \%$ & & 2.71828 & 1.40609449 & 0.34 & 0.874 & 0.807800 & 0.0090 & 0.000000 & $2,881,377$ \\
\hline \begin{tabular}{|l|l|}
56 & CAMESA \\
\end{tabular} & $4,031,386$ & 573,942 & 0.1121 & 789,298 & $5.30 \%$ & $46.36 \%$ & & 2.71828 & 5.10755591 & 1.63 & 2.112 & 0.974400 & 1.3091 & & $3,339,140$ \\
\hline \begin{tabular}{l|l|l|}
57 & INDUSTRIAS CHB \\
\end{tabular} & $5,017,366$ & 200,695 & 0.0000 & 200,695 & $5.30 \%$ & $30.40 \%$ & & 2.71828 & 25 & 3.22 & 3.516 & 0.999780 & 2.9900 & 0.998600 & $4,845,310$ \\
\hline \begin{tabular}{|l|l|}
58 & GMEXICO \\
\end{tabular} & $83,519,555$ & $27,458,863$ & 0.0637 & $33,049,736$ & $5.30 \%$ & $46.36 \%$ & & 2.71828 & 2.52708693 & 0.93 & 1.408 & & 0.6055 & & $55,983,887$ \\
\hline \begin{tabular}{|l|l|l|}
59 & PENOLES \\
\end{tabular} & $17,306,000$ & $4,696,158$ & 0.0450 & $5,359,544$ & 5.3 & $46.36 \%$ & & 2.71828 & 3.2290058 & 1.1 & 1.654 & 0.942900 & 0.8506 & 2300 & $12,741,408$ \\
\hline \begin{tabular}{l|l|l|}
60 & VITRO \\
\end{tabular} & $32,447,000$ & $15,879,697$ & 0.0883 & $20,471,264$ & $5.30 \%$ & $43.19 \%$ & & 2.71828 & 1.58500227 & 0.46 & 0.899 & 0.813300 & 0.1513 & 0.059600 & $25,348,415$ \\
\hline \begin{tabular}{l|l|}
61 & TEKCHEM \\
\end{tabular} & 724,613 & 138,312 & 0.0972 & 182,689 & $5.30 \%$ & $46.36 \%$ & & 2.71828 & 3.96637928 & 1.38 & 1.859 & 0.957300 & 1.0563 & 0.821200 & 565,702 \\
\hline \begin{tabular}{l|l}
62 & MEXICHEM S.A. \\
\end{tabular} & $2,139,919$ & 145,372 & 0.0414 & $\begin{array}{ll}164,178 \\
\end{array}$ & $5.30 \%$ & $46.36 \%$ & & 2.71828 & 13.0341006 & 2.57 & 3.049 & & 2.2460 & 0.985400 & $1,998,924$ \\
\hline \begin{tabular}{|l|l|}
63 & BANAMEX \\
\end{tabular} & $334,089,890$ & $281,446,550$ & 0.1003 & \begin{tabular}{|l|}
$374,912,519$ \\
\end{tabular} & 5.30 & $53.55 \%$ & & 2.71828 & 0.89111425 & -0.12 & 0.474 & 0.680800 & -0.4537 & 0.173600 & $171,931,282$ \\
\hline 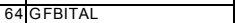 & $118,431,620$ & $107,066,780$ & 0.1165 & $149,010,529$ & $5.30 \%$ & $53.55 \%$ & & 2.71828 & 0.79478692 & -0.23 & 0.359 & 0.636800 & -0.5681 & 0.212300 & $48,432,768$ \\
\hline \begin{tabular}{|l|l|l|}
65 & FBB VA BANCOMER \\
\end{tabular} & $390,728,750$ & $349,459,390$ & 0.0995 & $464,453,286$ & $5.30 \%$ & $58.31 \%$ & & 2.71828 & 0.84126598 & -0.17 & 0.496 & 0.687900 & -0.5138 & 0.195000 & $191,527,791$ \\
\hline $\begin{array}{ll}66 \\
6\end{array}$ & $44,426,240$ & $19,483,180$ & 0.1667 & \begin{tabular}{|l|}
$30,943,035$ \\
\end{tabular} & 5.3 & & & 2.71828 & 1.4357428 & 0.3 & 0.674 & 0.74 & 0.1204 & 0.547800 & $18,798,686$ \\
\hline \begin{tabular}{l|l|l}
67 & GF BANORTE \\
\end{tabular} & $99,124,730$ & $90,860,800$ & 0.1142 & $125,680,896$ & $5.30 \%$ & $55.83 \%$ & & 2.71828 & 0.78870165 & -0.24 & 0.389 & 0.648000 & -0.5778 & 0.215700 & $41,108,629$ \\
\hline \begin{tabular}{l|l}
68 & AEROP. SURESTE
\end{tabular} & $10,382,658$ & 18,521 & 0.0356 & 20,569 & $5.30 \%$ & $65.42 \%$ & & 2.71828 & 504.762687 & 6.22 & 7.025 & 1.000000 & 5.8919 & 1.000000 & $10,365,112$ \\
\hline 69 MEDICA SUR & $1,010,400$ & 197,847 & 0.0403 & 222,774 & & $65.42 \%$ & & 2.71828 & 4.53553445 & 1.51 & 2.313 & & 1.1798 & 0.868600 & 833,017 \\
\hline \begin{tabular}{l|l|l}
70 & TAMSA \\
\end{tabular} & $11,888,162$ & $1,305,810$ & 0.0676 & $1,588,998$ & $5.30 \%$ & $51.50 \%$ & & 2.71828 & 7.48154744 & 2.01 & 2.569 & 0.993200 & 1.6773 & 0.942900 & $10,529,307$ \\
\hline \begin{tabular}{l|l|}
71 HYLSAMEX \\
\end{tabular} & $29,904,314$ & $14,061,493$ & 0.0760 & $17,517,325$ & $5.30 \%$ & $48.25 \%$ & & 2.71828 & 1.70712784 & 0.53 & 1.043 & 0.823800 & 0.2073 & \begin{tabular}{|l|}
0.535900 \\
\end{tabular} & $16,627,640$ \\
\hline
\end{tabular}

Notas :

Activo Total : Datos tomados del Anuario Financiero del año 2000 publicado por la Bolsa Mexicana de Valores

2 Pasivo : Pasivo con costo, dato tomado del Anuario Financioro delano 2000 publicado por la la Bolsa Mexicana de Valores

${ }^{3}$ Costo del Pasivo : Se calculó así : (Intereses pagados/Pasivo con Costo) - Tasa Fiscal

${ }^{4}$ Pasivo a futuro: Se calculó de acuerdo con los requerimientos del modelo : Como pasivo con bono cupón cero : Pasivo (1+ costo del pasivo)

${ }^{5}$ Se calculó asi : :"( $1+$ Tasa Nominal de Cetes $) /(1+$ Tasa de Inflación $\left.)\right)-1$

${ }^{6}$ Volatilidad : Dato tomado del anuario bursátil 2000, publicado por la Bolsa Mexicana de Valore

Tiempo a vencimiento : Se consideran 3 años debido a que las empresas mexicanas por lo general no se endeudan a largo plazo.

e : es una constante matemática

${ }^{9} \mathrm{~S} / \mathrm{X}$ : Es el activo entre el pasivo

Log. Natural : Es el logaritmo natural del activo entre el pasivo

d 1 : de acuerdo con la fórmula del modelo de Black y Scholes

${ }^{12}$ Normal : Es el valor del área bajo la curva normal

${ }^{13} \mathrm{~d} 2$ : de acuerdo con la fórmula del modelo de Black y Scholes

${ }^{14} \mathrm{C}$ : Este resultado es el valor de la empresa de acuerdo con el modelo de Black y Scholes

Fórmula del Modelo de Black y Scholes :

$C=S N\left(d_{1}\right) \cdot X e^{-r 1} N\left(d_{2}\right)$

$$
\begin{aligned}
& d_{1}=\frac{\ln (S / X)+\left(r+\eta^{2} / 2\right) T}{2 ? T} \\
& d_{2}=d_{1}-2 ? T
\end{aligned}
$$

$\mathrm{C}=$ Precio de la opción/ Valor de la empresa

$S=$ Precio del activo suby acente/ Valor presente de los flujos de efectivo
$X=$ Precio de ejercicio/ Valor futuro de la deuda

= Volatilidad del precio del activo/ Volatilidad de los flujos de efectivo

The

1n $=$ Logaritmo natural

$\mathrm{N}\left(\mathrm{d}_{\mathrm{t}}\right)=\mathrm{Func}$ ción de densidad del área bajo la curva normal estándar 


\begin{tabular}{|c|c|c|c|c|c|c|c|c|c|c|c|}
\hline \multicolumn{12}{|c|}{ Anexo 2} \\
\hline \multirow{2}{*}{\multicolumn{12}{|c|}{ Cálculo del valor promedio sectorial del modelo Black y Scholes (1991-2000) }} \\
\hline & & & & & & & & & & & \\
\hline \multicolumn{12}{|c|}{ Empresas que cotizan en la Bolsa Mexicana de Valores } \\
\hline & & \multirow{2}{*}{\multicolumn{5}{|c|}{ (Miles de pesos) }} & & & & & \\
\hline \multicolumn{2}{|c|}{ Alimentos, Bebidas y Tabaco } & & & & & & & & & & \\
\hline \begin{tabular}{l|l|l|l|l} 
No & Sector \\
\end{tabular} & Empresa & 1991 & 1992 & 1993 & 1994 & 1995 & 1996 & 1997 & 1998 & 1999 & 2000 \\
\hline 1|Alimentos & BIMBO & $11,616,031$ & $11,928,273$ & $13,144,301$ & $16,370,893$ & $16,747,038$ & $14,589,435$ & $16,311,384$ & $17,426,113$ & $18,988,456$ & $20,182,586$ \\
\hline 2 Alimentos & CONTINENTAL GPO. & $4,198,325$ & $4,167,503$ & $4,075,091]$ & $5,088,376$ & $3,719,400$ & $4,036,668$ & $5,012,895$ & $5,867,935 \mid$ & $6,829,471$ & $7,761,625$ \\
\hline 3 Alimentos & FEMSA & $18,148,258 \mid$ & $20,712,624 \mid$ & $22,457,878 \mid$ & $32,696,782$ & 31,107,364] & $24,564,218$ & $28,024,483 \mid$ & $31,350,412 \mid$ & $33,046,470$ & $35,932,549$ \\
\hline 4 Alimentos & GMASECA & n.d. & $6,000,291 \mid$ & $6,395,241 \mid$ & $11,468,718$ & 10,618,835 & $11,800,072$ & 14,145,247| & $12,235,821]$ & $13,485,137$ & $14,072,061$ \\
\hline \begin{tabular}{|l|l|l|}
5 Alimentos \\
\end{tabular} & GMODELO & $24,009,006$ & $26,533,769 \mid$ & $28,973,645 \mid$ & $35,209,284$ & $34,441,159 \mid$ & $34,245,762$ & $37,506,076 \mid$ & $39,320,710$ & $40,075,406$ & $45,576,831$ \\
\hline \begin{tabular}{|l|l|}
6 Alimentos \\
\end{tabular} & SAVIA & $4,057,698]$ & $5,508,104$ & $4,846,342$ & $14,149,072$ & 16,874,114 & $11,087,588$ & $23,092,955$ & $19,938,283 \mid$ & $45,967,122$ & $48,100,465$ \\
\hline 7 Alimentos & BACHOCO & n.d. & \begin{tabular}{l|l} 
n.d. & \\
\end{tabular} & n.d. & n.d. & $4,578,144$ & $4,634,207$ & $|6,145,963|$ & $|6,882,661|$ & $7,7339,764$ & $8,800,028$ \\
\hline $8 \mid$ Alimentos & GEM UNIDAS & $1,008,518$ & $1,470,661]$ & $2,088,739$ & $2,399,257$ & $1,945,878$ & $1,910,778$ & $2,079,634 \mid$ & $|1,671,451|$ & $1,661,322$ & $1,864,256$ \\
\hline \begin{tabular}{l|l|l|l|l}
9 & Alimentos
\end{tabular} & GMODERNA & $1,091,206$ & $\mid 1,378,828$ & $\mid 1,485,754$ & $2,207,762$ & $1,948,821$ & $1,975,985$ & $\mid 1,904,146$ & $1,998,391 \mid$ & \begin{tabular}{|l|l|l|}
$, 968,127$ \\
\end{tabular} & $2,206,472$ \\
\hline 10|Alimentos & ARGOS & \begin{tabular}{l|l} 
n.d. & \\
\end{tabular} & \begin{tabular}{l|l} 
n.d. & \\
\end{tabular} & n.d. & & \begin{tabular}{l|l} 
n.d. \\
\end{tabular} & n.d. & \begin{tabular}{l|l} 
n.d. & \\
\end{tabular} & $2,948,456$ & $2,286,965$ & $3,110,800$ \\
\hline 11|Alimentos & HERDEZ, S.A. & $1,287,012$ & $1,741,058$ & $1,844,796$ & $2,186,120$ & $1,457,461]$ & $1,640,693$ & $1,924,477$ & $1,885,329$ & $2,167,633$ & $2,679,183$ \\
\hline 12|Alimentos & |MAIZORO & \begin{tabular}{l|l} 
n.d. & \\
\end{tabular} & \begin{tabular}{l|l} 
n.d. & \\
\end{tabular} & n.d & n.d. & 375,210 & 295,619 & 327,463 & 286,896 & 311,384 & 316,171 \\
\hline \begin{tabular}{|l|l|}
13 & Alimentos \\
\end{tabular} & KOF COCA COLA & $\begin{array}{l}\text { n.d. } \\
\text { s }\end{array}$ & $\mid 1,673,720$ & $3,016,029$ & $7,713,382$ & $7,143,117$ & $\mid 6,792,664$ & 7,176,177| & $8,079,817$ & $8,218,068$ & $8,622,705$ \\
\hline 14/Alimentos & GMASECA & $2,469,785$ & $3,270,501$ & $4,115,900$ & $5,896,246$ & $6,174,623$ & 6,325,320 & $\mid 6,491,142$ & $6,707,725$ & $6,725,521$ & $6,738,003$ \\
\hline & Suma & $67,885,839$ & $84,385,332$ & $92,443,715$ & $135,385,891$ & $\begin{array}{l}137,131,166 \\
\end{array}$ & $\overline{123,899,010}$ & $150,142,043$ & $156,599,999$ & $189,570,845$ & $205,963,733$ \\
\hline & Promedio & $\begin{array}{l}7,542,871 \\
\end{array}$ & $\mid 7,671,394$ & $\mid 8,403,974$ & $12,307,808$ & $\mid 10,548,551$ & 9,530,693 & $\mid \begin{array}{l}11,549,388 \\
\end{array}$ & 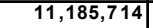 & $13,540,775$ & $\begin{array}{l}14,711,695 \\
\end{array}$ \\
\hline \multirow{2}{*}{$\begin{array}{ccc}\text { Comercio } \\
\text { Nolsoctor }\end{array}$} & & & & & & & & & & & \\
\hline & Empresa & 1991 & 1992 & 1993 & 1994 & 1995 & 1996 & 1997 & 1998 & 1999 & 2000 \\
\hline 1/Comercio & COM. MEXICANA & $10,818,898$ & $12,860,476$ & $14,682,169$ | & $15,592,829$ & $13,247,147$ & $13,385,227$ & $15,655,369$ & $16,410,659 \mid$ & $17,764,901$ & $18,518,138$ \\
\hline \begin{tabular}{l|l}
2 & Comercio
\end{tabular} & ELEKTRA & $4,820,527$ & $5,351,236$ & $5,683,959$ & $10,612,089$ & 9,417,706 & 9,740,238 & $\mid 11,413,672$ & $11,556,976$ & $12,325,417$ & $17,207,142$ \\
\hline \begin{tabular}{|l|}
3 \\
Comercio
\end{tabular} & SORIANA & $1,492,643$ & $1,960,639$ & $2,494,966$ & $3,528,145$ & $4,276,399$ & 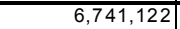 & $\mid 11,764,393$ & \begin{tabular}{|c|}
$9,313,595$ \\
\end{tabular} & 10,317. & $8,271,141$ \\
\hline 4 Comercio & WALL MART MEX & 19,536,656 & $26,767,441$ & $35,226,365$ & $36,313,681$ & $33,893,941$ & $32,937,404$ & $43,237,031$ & $43,407,653$ & $46,129,253$ & $50,262,308$ \\
\hline \begin{tabular}{r|c|}
5 & Comercio
\end{tabular} & SABA CASA GPO. & $2,347,742$ & $\mid 1,809,789$ & $3,259,196$ & $3,615,358$ & $3,556,749$ & \begin{tabular}{|l|l|}
$3,592,989$ \\
\end{tabular} & $4,025,052$ & $\mid 4,216,571$ & $3,797,504$ & $6,059,982$ \\
\hline 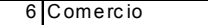 & DATAFLUX & & & & & 650,158 & 848,383 & $1,587,520$ & $1,253,353$ & 839,951 & 993,108 \\
\hline \begin{tabular}{|l|l|}
7 Comercio \\
\end{tabular} & EDOARDO & 356,544 & 333,147 & 363,402 & 437,569 & 419,583 & $\mid 411,354$ & $\mid 524,248$ & | & 616,651 & 593,461 \\
\hline 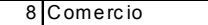 & FRAGUA CORP. & n.d. & n.d. & n.d. & $\begin{array}{ll}\text { n.d. } \\
\end{array}$ & 574,599 & 483,773 & 889,969 & $1,097,869$ & $1,499,712$ & $1,614,850$ \\
\hline \begin{tabular}{|l|l|}
9 & Comercio \\
\end{tabular} & GCORVI & $\begin{array}{l}\text { n.d. } \\
\text { nat }\end{array}$ & n.d. & n.d. & n.d. & $1,575,172$ & $2,217,839$ & $2,376,709$ & $2,684,395$ & $3,092,866$ & $3,339,957$ \\
\hline $\begin{array}{l}10 / \text { Comercio } \\
\end{array}$ & GIGANTE & 11,381,174] & 12,091,635 & 16,617,170 & $17,717,244$ & $\overline{13,007,864}$ & $15,535,756$ & $15,650,615$ & 16,322,730 & $\frac{16,982,936}{}$ & $16,520,889$ \\
\hline $\begin{array}{l}11 \text { Comercio } \\
\end{array}$ & GPALACIO HIERRO & $4,324,812$ & \begin{tabular}{|l|l}
$4,681,029$ \\
\end{tabular} & $5,274,880$ & $7,057,357$ & $5,451,688$ & $4,827,156$ & 5,486,775 & $5,987,231[$ & $5,228,430$ & $6,942,300$ \\
\hline \begin{tabular}{|l|l|}
12 Comercio \\
\end{tabular} & ALSEA & & & & & & & & 611,783 & 894,589 & $1,164,513$ \\
\hline 13|Comercio & NADRO & $1,467,194$ & $1,575,860$ & $1,921,768$ & $2,747,279$ & $3,043,974$ & 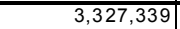 & $3,783,796$ & $4,346,156$ & $4,740,992$ & $4,128,022$ \\
\hline 14 Comercio & LIVERPOOL & n.d. & \begin{tabular}{l|l} 
n.d. \\
\end{tabular} & $\begin{array}{ll}\text { n.d. } \\
\end{array}$ & n.d. & 14,564,041] & $9,270,604$ & $\mid 11,367,989$ & $\mid 11,307,809$ & $12,433,645$ & $14,118,022$ \\
\hline \begin{tabular}{c|c|}
15 & Comercio \\
\end{tabular} & GPROVE QUIM & & n.d. & & n.d. & 239,431 & 197,272 & 322,221 & 387,625 & 431,778 & 312,042 \\
\hline & Suma & $56,546,190$ & $67,431,251$ & 23,876 & $97,621,551$ & $103,918,451$ & $103,516,456$ & $128,085,359$ & $129,512,867$ & $137,488,945$ & $150,045,875$ \\
\hline & Promedio & $6,282,910$ & $7,492,361$ & $9,502,653$ & $10,846,839$ & $7,422,746$ & $7,394,033$ & $\mid 9,148,954$ & $8,634,191$ & $9,165,930$ & $\begin{array}{l}10,003,058 \\
\end{array}$ \\
\hline \multirow{2}{*}{\begin{tabular}{|l} 
Comunic aciones y T \\
No|Sector
\end{tabular}} & & & & & & & & & & & \\
\hline & ransportes & 1991 & 1992 & 1993 & 1994 & 1995 & 1996 & & & & \\
\hline & Empresa & 1991 & 1992 & 1993 & 1994 & 1995 & 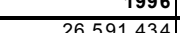 & 年 & 1998 & 1999 & 2000 \\
\hline $\begin{array}{l}1 \text { Comunicaciones } \\
2 \text { Comunicaciones }\end{array}$ & TELEVISA & $\frac{19.925,763}{19.93}$ & $21,778,455$ & 27.480 .501 & $34,412,632$ & $37,322,887$ & $20,0,09,4,454$ & $\mid \frac{2,20,2,0,1}{36,212,872}$ & 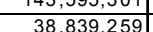 & $\begin{array}{l}30,450,000 \\
33,506,251\end{array}$ & $\frac{100,361,038}{34,324,269}$ \\
\hline \begin{tabular}{l|l}
3 & Comuni
\end{tabular} & TELMEX & $65,163,440$ & $77,163,145$ & $97,479,352$ & $134,814,261$ & $108,871,845$ & $97,069,817$ & $78,224,834$ & $86,320,345$ & $134,003,976$ & $92,374,914$ \\
\hline $4 \mid$ Comur & TVAZTECA & & & & & & & & & & $13,195,536$ \\
\hline 5 Comunicaciones & TMM & $3,206,416$ & $\mid 4,173,887$ & $7,969,746$ & $7,985,444$ & $8,737,295$ & $5,963,986$ & $7,292,083$ & $11,233,141]$ & $8,952,856$ & $17,494,686$ \\
\hline $\begin{array}{l}6 \\
6\end{array}$ & SINTRA & \begin{tabular}{l|l} 
n.d. & \\
\end{tabular} & $\begin{array}{ll}\text { n.d. } \\
\end{array}$ & \begin{tabular}{l|l} 
n.d. & \\
\end{tabular} & $\begin{array}{ll}\text { n.d. } \\
\end{array}$ & 12,14 & $8,992,690$ & $\mid 12,622,436$ & $|3,858,928|$ & 13,82 & $15,551,974$ \\
\hline 7 Comu & RAD & $\begin{array}{l}\text { n.d. } \\
\text {. }\end{array}$ & 650,997 & 936,866 & $1,344,068$ & & & & & & $\begin{array}{ll}1,449,287 \\
\end{array}$ \\
\hline 8 Comu & MOVIL ACCES & n.d. & \begin{tabular}{l|l} 
n.d. & \\
\end{tabular} & & & & & 506,089 & & & 546,005 \\
\hline 9 Comunicaciones & $\begin{array}{l}\text { SIUSACELL } \\
\end{array}$ & n.d. & $2,009,499$ & & $9,885,423$ & & & $5,962,299$ & & & $10,245,206$ \\
\hline & Suma & $88,295,620$ & $105,775,983]$ & $139,303,715$ & $188,441,827$ & $183,097,284$ & $179,015,422$ & 172,824,816 & $313,933,796$ & $348,312,972$ & $345,542,915$ \\
\hline & Promedio & 29,431,873 & $21,155,197$ & \begin{tabular}{|l|l|}
$27,860,743$ \\
\end{tabular} & $37,688,365$ & $22,887,161$ & $19,890,602$ & 19,202,757 & $34,881,533$ & $38,701,441$ & $38,393,657$ \\
\hline Co & & & & & & & & & & & \\
\hline $\begin{array}{l}\text { Construction } \\
\text { No|Sector }\end{array}$ & Empresa & 1991 & 1992 & 1993 & 1994 & 19955 & 1999 & 1997 & 1009 & 10000 & \\
\hline No pector & Empresa & 19914 & $\begin{aligned} 91992 \\
\end{aligned}$ & $\frac{19934}{1029}$ & $\frac{1944}{12590}$ & (1955) & 1996 & $\frac{19291}{1290}$ & 19980 & 1999 & 2000 \\
\hline $\begin{array}{l}1 \text { Construccion } \\
2 \text { Construcción }\end{array}$ & CEMMENTOS CHIHUAHU & $\frac{8,220,333}{2035975}$ & $\begin{array}{l}9,482,103 \\
3069,152 \\
\end{array}$ & $\mid 0,110,444$ & $\frac{10,010,290}{3908,470}$ & & $\frac{2,201}{3033}$ & $\mid \frac{\mid, 2,27,040}{3717,108}$ & $\frac{12,985,9}{40915}$ & (1,0030 & $\frac{13,567,431}{3654416}$ \\
\hline 3 Construc & $\begin{array}{l}\text { CEMEX S A } \\
\end{array}$ & 23,765731 & $\frac{0,005,152}{47,601,371}$ & $\frac{1,07,047}{42,640,053}$ & $\frac{0,950,410}{71,602,028}$ & & & & $\frac{4,015,}{70,140}$ & & $\begin{array}{r}3,654,416 \\
94,436,646\end{array}$ \\
\hline Alconstruc & OEDAN, S.A. & 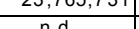 & 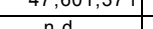 & $42,04,0,030$ & $\frac{11,00,0,020}{4}$ & & & 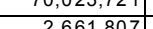 & 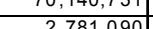 & $\frac{\mid 0,30,0.053}{3,569}$ & 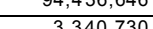 \\
\hline 4 [|Construcc & ARA & n.d. & n.d. & & & & & $2,661,807$ & $2,781,090$ & $3,456,159$ & \\
\hline 5 [ onstruccion & GEO & 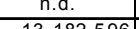 & $\begin{array}{l}\text { n.c. } \\
1 \text {. }\end{array}$ & $\begin{aligned} 548,638 \\
250\end{aligned}$ & $\frac{1,696,133}{2,277}$ & 20,419,306 & $\frac{2,208,415}{2,201}$ & |3,63, & 年, & $\frac{3,57,286}{17,921}$ & $\begin{array}{r}3,953,892 \\
16,20,205\end{array}$ \\
\hline 6 Construcción & ICA SOC. CONTROL. & $13,182,596$ & $16,540,4800$ & $23,563,288$ & $\frac{32,220,577}{120,07,107}$ & $30,397,5666$ & $21,498,981$ & $\begin{array}{r}22,689,219 \\
14,427\end{array}$ & $27,327,423\}$ & $17,489,034$ & $16,326,395$ \\
\hline & Suma & 47,204,635 & $76,693,106]$ & $80,245,594$ & 123,027,497 & $140,968,508$ & $95,415,724$ & $114,942,674$ & $121,466,080$ & $111,753,548$ & $135,279,509$ \\
\hline & Promedio & 11,801,159| & $19,173,276$ & $16,049,119$ & $24,605,499$ & 23,494,751 & $15,902,621$ & 19,157,112 & $20,244,347$ & 18,625,591 & $22,546,585$ \\
\hline n.d. No hubo datos di & isponibles para realizar & aalculc & & & & & & & & & \\
\hline
\end{tabular}

